\title{
Grain Orientation Dependence of Phase Transformation in the Shape Memory Alloy Nickel-Titanium
}

\author{
Michael Kimiecik ${ }^{\mathrm{a}}$, J. Wayne Jones ${ }^{\mathrm{a}}$, Samantha Daly, \\ ${ }^{\mathrm{a}}$ Department of Materials Science and Engineering, University of Michigan, 2300 Hayward, Ann Arbor, MI 48109, \\ USA \\ ${ }^{\mathrm{b}}$ Department of Mechanical Engineering, University of Michigan, 2350 Hayward, Ann Arbor, MI 48109, USA
}

The dependence of grain orientation on stress-induced martensitic transformation in superelastic, polycrystalline Nickel-Titanium sheet was examined at the microstructural length scale. Fullfield strains, indicative of transformation extent, were characterized in fields of view of nominally $100 \mu \mathrm{m}$ x $100 \mu \mathrm{m}$ using a custom combination of scanning electron microscopy with distortion-corrected digital image correlation. It was found that similarly oriented grains do not necessarily transform similarly, in contrast to a common assumption in mean-field theories. Specifically, grains with similar orientation (as determined by the misorientation of the grain and specimen axes) showed variation in both the mean strain of the grain as well as the range (heterogeneity) of strain across the grain, as determined from surface measurements. Additionally, neither grain size nor degree of misorientation (of common crystal axes from the loading axis) affected the mean strain and strain range.

Keywords: Nickel Titanium, Superelasticity, Scanning Electron Microscopy, Digital Image Correlation, Mean Field Theory

\footnotetext{
* Corresponding Author: Department of Mechanical Engineering, University of Michigan, 2350 Hayward, Ann Arbor, MI 48109-2125, USA, e-mail: samdaly@umich.edu , phone: 1-734-763-8137
} 


\section{Introduction}

Nickel-Titanium (NiTi) alloys belong to a class of materials commonly referred to as shape memory alloys (SMAs). The defining characteristic of SMAs is the ability to recover strains as large as $10 \%$ through a solid-to-solid, diffusionless phase transformation [1]. In the case of NiTi, this transformation takes place between a high symmetry cubic (B2) austenite and one or more monoclinic $\left(\mathrm{B} 19^{\prime}\right)$ martensite variants [1-4]. While the crystallography and thermomechanical properties of this transformation are well understood for single crystals [2,5-7], the mechanisms of transformation are unclear in polycrystalline SMAs. Mean-field and phenomenological models of phase transformation [8-15] use properties derived from observing single crystal behavior and assumptions about the similarity and completeness of martensite transformation in grains of similar orientation to predict macroscopic stress-strain curves of polycrystalline SMAs. While these predictions are reasonable approximations of averaged macroscopic behavior under simple loading conditions, their underlying assumptions have not been validated and may not be widely applicable. Futhermore, recent theoretical work suggests that martensitic transformation in polycrystals can take on complex and heterogeneous configurations when the constraints of neighboring grains and interactions with other deformation mechanisms (such as plasticity) are taken into account [16]. The aim of this work is to examine the assumptions used in many meanfield theories that grain orientation is sufficient to predict the magnitude of martensite transformation strain and that grains transform fully.

Transformation strain and habit plane formation in NiTi single crystals have been identified experimentally [2,7,17-19] and modeled using phenomenological theory of martensite transformation [13,20,21] for both tensile and compressive loading. Under tensile loading $[2,7,17]$, the experimentally observed transformation strain was consistent with a single 
correspondent variant pair (CVP) completely replacing the parent austenite volume at the end of the transformation plateau. Additionally, martensitic variants that dominated the transformation were identified by the delineation of specific habit planes on the sample surface $[2,4,17]$ and matched well with predictions based on the Schmid factor for each variant. For a single crystal of Nickel-Titanium under uniaxial tension, the total strain accommodated by the martensite for an arbitrary crystal orientation could be accurately predicted [2,22]. Characterization of single crystals under compression [6,19] has also been performed. However, because non-local transformation of bulk NiTi occurs under compression, no defined habit plane is formed, making macroscopic identification of the particular active CVPs difficult. Rather, these studies focused on the critical resolved shear stress required for martensitic transformation and on the relative activity of plastic deformation and transformation. For many grain orientations, a similar level of plastic deformation and martensitic transformation under compression made attribution of measured strain to one of these mechanisms more difficult than in the simple tension case, where the stress required for transformation is well below that required for plastic deformation [19]. However, in grains with orientations where plasticity was significantly suppressed, compression results provided confirmation of transformation strains predicted by the phenomenological model for single crystals. More recently, single crystal experiments performed on micropillars in compression have allowed for the confirmation of exact martensite variants active under compressive loads [23-27]. At the microscale bulk plasticity is suppressed, and in solutionized samples individual CVPs can form with distinct and measureable habit planes [23]. Under these conditions the formation of specific martensite variants can be determined by habit plane measurement and the results can be compared directly against phenomenological predictions [22]. When combined with bulk single crystal results, the confirmation of specific active CVPs 
in micro compression validates the predictive power of phenomenological models for uniaxial loading of single crystals of arbitrary orientation.

The results of single crystal experiments are used to extend predictions of transformation strain and martensite variant selection to polycrystalline NiTi $[14,21,28-31]$. In polycrystalline $\mathrm{NiTi}$, the constraints imposed by neighboring grains complicate the stress states of individual grains, which can no longer be described by the uniform global stress state. One approximation used to circumvent the complications of such constraints is to consider a polycrystalline solid as an averaged collection of single crystals, with orientations determined by the orientation distribution function (ODF) [21,29,30,32]. An estimate of the total recoverable strain for shape memory [30] or superelasticity $[21,29,32]$ can then be made by averaging the contribution of each independent austenite grain by assuming complete transformation to martensite. By choosing the most favorable single variant of martensite, this averaging produces an upper bound that can overestimate the bulk strain in polycrystalline samples by as much as $100 \%$ of the experimentally measured strain $[29,32]$. However, this approach does capture trends, such as the decrease of maximum accommodated strain in textured, rolled material when tensile deformation occurs perpendicular rather than parallel to the rolling direction. An improved estimate can be made by considering the most favorable $\mathrm{CVP}$ - rather than the individual variant—of martensite $[22,29]$. While this calculation better matches the recovered strain magnitude, the variation in transformation strain shows less sensitivity to sample orientation and is still an overestimate that ignores the constraints imposed by neighboring grains when compared with experimental data. A further refinement can be made by considering the Taylor bound of accommodated strains for single crystals, in which multiple CVPs of martensite are allowable [29,31,33]. This last consideration includes compatibility requirements at representative grain boundaries and most 
closely matches experiment, but again much of the orientation dependence is lost, resulting in an underestimate of the recoverable strains in polycrystalline specimens.

Although the incorporation of incomplete transformation and alternate deformation modes such as bulk plasticity and dislocation motion have been proposed to improve model accuracy, the underlying assumptions of applying single crystal response to polycrystals requires further investigation. Combined single crystal responses do not accurately predict the transformation strain of polycrystalline specimens. Phenomenological models assume a single transformation path—of either a single variant, variant pair, or variant subset—to consume the entirety of a crystal oriented along a particular axis. Measuring the details of this phase change directly is difficult because of the small length scale of the twinned martensite microstructure. However, each transformation path imparts a known strain based on the phase fraction of each martensite variant in the final configuration. While it is possible for different transformations to accommodate the same strain, a particular martensite volume fraction/configuration pair cannot simultaneously maintain different strain levels. Thus, while strain measurement alone cannot confirm that similarly oriented grains have transformed to the same configuration of martensite or to the same volume fraction of the same variants, it can pinpoint at what strain and the specific locations (grains) where different martensite configurations form.

In the present study, an investigation of a principal assumption in most mean-field and phenomenological theories-that similarly oriented grains in polycrystalline SMAs transform similarly—was conducted using structural information from electron backscattered diffraction (EBSD) linked to local strain data provided by digital image correlation of scanning electron micrographs (SEM-DIC). This approach enabled grain-to-grain comparisons of transformation strain and grain orientation which satisfied three principal criteria: First, that strain, phase, and 
orientation data can be quantitatively compared, either within the same measurement or between multiple measurements, to associate microstructural features with martensite transformation and recovery. Second, that the approach has sufficient resolution relative to the microstructural features (e.g. parent austenite grains) to allow multiple aspects of the martensite transformation (i.e. overall transformation strain and transformation heterogeneity) to be compared across the sampled microstructure. Lastly, that a single measurement captures strain (or phase) data equally well for martensite and austenite phases. This last requirement is difficult to address experimentally, given the disparate size scales of parent austenite and martensite microstructures. Previous studies using diffraction-based techniques [34,35] were the first to achieve the necessary resolution to examine martensitic transformation on the microstructural length scale. However, the fineness of martensite twins required that martensite transformation be inferred from the absence of austenite diffraction patterns. Additionally, local strain measurements were limited to the elastic strain of the austenite phase without a similar local measurement of transformation strain in martensite. Optical microscopy [36] has been successfully used to resolve the fine martensite surface structure, but did not link this data to local strains. The experimental approach used here tracked the local strains of both phases with high spatial resolution and accuracy across large fields of view, in order to determine if similarly oriented grains do or do not transform similarly, with regard to either their mean strain or the range of strain they accommodate.

\section{Experimental}

\subsection{Strain Measurement}

The use of scanning electron microscopy paired with digital image correlation (SEM-DIC) to measure full-field surface displacements was chosen because it provided the necessary high- 
resolution, high accuracy data across a large field of view relative to the microstructure in order to extract a statistically significant analysis of surface deformation behavior [37-39]. Highresolution SEM imaging combined with digital image correlation, an optical deformation tracking methodology, allowed strains associated with small microstructural features to be resolved over a relatively large statistically significant area. In this study, each strain field encompassed approximately 5000 grains over a nominally $100 \mu \mathrm{m} \times 100 \mu \mathrm{m}$ imaging area. Fullfield displacements (and thus Lagrangian strains) were calculated using DIC, which functions by tracking image subsets between reference and deformed image pairs [40,41]. Subsets are matched between images by minimizing a cross-correlation coefficient, which often requires the application of a high contrast, uniformly distributed pattern to the sample surface. This pattern was applied through the use of chemically functionalized nanoparticles, as described in section 2.2. The local displacement field produced by this subset tracking was then used to calculate the local deformation gradient and in-plane strain of the sample surface. Full-field strains from each image were subsequently aligned with the underlying microstructural data for analysis.

\subsection{Material and Sample Preparation}

Superelastic SE508 NiTi sheet with an initial thickness of $0.480 \mathrm{~mm}$ was obtained from Nitinol Devices and Components. Dogbone-shaped specimens were cut using electro-discharge machining, with the tensile loading axes parallel to the sheet rolling direction. Specimen dimensions are shown in Figure 1a. Specimens were held at $625^{\circ} \mathrm{C}$ for 10 minutes and then annealed at $450^{\circ} \mathrm{C}$ for 10 minutes $[42,43]$ to produce an equiaxed microstructure with an typical grain diameter of $5 \mu \mathrm{m}$ as determined by the area average of all grains. The sample discussed in Section 3 has a slightly smaller average diameter of $3.65 \mu \mathrm{m}$. 
Samples were manually ground to a thickness of approximately $300 \mu \mathrm{m}$ and polished to a mirror surface, with a final polishing step of vibratory polishing in colloidal silica. Platinum markers were then deposited on each sample to delineate a nominally $100 \mu \mathrm{m} \times 100 \mu \mathrm{m}$ imaging area and for later use in aligning the crystallographic (EBSD) and strain (SEM-DIC) data sets. Electron backscatter diffraction (EBSD) was used to map grain boundary locations and orientations of individual austenite grains. The EBSD scan of this sample is shown in Figure $1 \mathrm{~b}$. EBSD scans were performed with a $0.2 \mu \mathrm{m}$ step size at 20 frames per second and cleaning of the EBSD data resulted in exclusion of less than $5 \%$ of points, most of which were single point artifacts. A similar scan could be produced using a wide range of scan rates and step sizes depending on surface finish and beam settings. It is important to note that the resolution of the EBSD scan is much lower than the 5.5 million point image ( $2033 \times 2716$ points) generated for strain measurement. A rolling texture of $\{1 \overline{1} 1\}[110]$ was largely preserved, albeit slightly weakened, through this recrystallization treatment. Samples were patterned for micro digital image correlation using a self-assembly of gold nanoparticles secured to the sample surface by a silane monolayer [44], as shown in Figure 1c. The smallest feature produced by this nanoparticle patterning technique was approximately $50 \mathrm{~nm}$, which translated into an image resolution of 15 $\mathrm{nm} /$ pixel. This resolution prevented the aliasing of individual nanoparticles by ensuring that the smallest traceable feature — a single nanoparticle—was at least $3 \times 3$ pixels in each image [40].

\subsection{Microscale Deformation Mapping}

Patterned specimens were tested in-SEM under uniaxial tension at room temperature using a microtensile heating-straining stage (Kammrath and Weiss $1 \mathrm{kN}$ Tensile/Compression Module), through one complete transformation cycle-from austenite to 'fully transformed' martensite and back to austenite. 'Fully transformed' martensite is defined by the complete propagation of 
localized bands of martensite through the test specimen and the corresponding completion of the stress plateau in the macroscopic stress-strain curve. However, note that 'fully transformed' martensite is not actually fully transformed, as residual areas of low strain, locked-in austenite remain (Figure 1d). Micrographs of the patterned surface were captured in situ throughout the loading cycle using a Tescan Mira 3 FEG SEM. Initial correlation was performed using commercial software [41] to obtain raw displacement data. This raw displacement data was then analyzed and corrected for image distortions using custom MATLAB scripts described in detail in [37-39]. Briefly, the distortion correction process consists of two major parts. First, known rigid body translations were captured in situ to correct the spatial aberrations inherent in the SEM imaging system. After the removal of spatial distortions, individual pixel drift (occurring during each image scan) was detected and corrected by comparing the displacement maps of static image pairs. These corrections are necessary to obtain accurate quantitative information from SEM-DIC.

Strain fields captured in this study were limited to measuring the 2-D in-plane surface strains that arose during deformation. It is possible that neglecting out-of-plane displacements, which could not be measured with the experimental setup used here, might influence the accuracy of inplane displacements. However, given that the samples were thin, planar specimens with no large stress concentrators (i.e. cracks) in the area of interest, it is likely that that any out-of-plane displacements of the sample surface were quite small. Subsurface microstructural features could also influence measured surface displacements. Models of other engineering materials that were calibrated against a full experimental 3-D microstructure had difficulty predicting surface strains when only surface orientation information was available [45]. In this work, the effects of subsurface microstructure were not taken into account. However, the phenomenological 
assumption that similarly oriented grains transform similarly, regardless of neighboring grains, should hold true for surface grains independent of their subsurface surroundings, if the assumption is indeed true. Analysis derived from surface measurements, such as the work presented here, is therefore sufficient to confirm or refute theoretical frameworks that do not take neighborhood effects into account.

\section{Results and Discussion}

In this section grain similarity is first defined. Following this, the relationship between the martensitic transformation of a grain and its crystallographic orientation is presented from analyses of the strain maps derived in the in-SEM DIC studies. Specific attention is paid to transformation extent and heterogeneity as they relate to grain orientation.

\subsection{Characterization of Grain Similarity}

Grains were categorized by the orientation of each austenite crystal along the loading axis of the sample. Two grains, G1 and G2, were designated as having the same crystallographic loading axis if a rigid body rotation of G1 about the sample loading axis matched the orientation of G2 after taking into account all symmetry operators (Figure 2$)$. A small range of deviation $\left(\theta \leq 5^{\circ}\right.$ or $2.5^{\circ}$ depending on orientation population) of loading orientations from a low index direction was used to bin grains into similarly oriented groups in the analysis, as perfect alignment was improbable in a real material. One benefit of this rotation-based definition for grain orientation similarity is the lack of any change to strain measurements along the rotation axis, which in this case was the loading axis. Preserving strain values along the loading axis by implementing this definition of grain similarity was necessary to directly compare experimental measurements of transformation strain with phenomenological predictions of martensitic transformation. Maps of the maximum strain predicted in single crystals with different loading axes can readily be found 
in the literature $[17,20,28]$ (Figure 3a). Direct comparison can be made between polycrystals, which are essentially comprised of constrained single crystals, and unconstrained single crystals. Note that comparing strains along axes other than the loading axis is not straightforward in these experiments. Rotations about the loading axis can align off-axis orientations, but DIC-obtained strain measurements cannot be similarly reoriented. Two-dimensional SEM-DIC cannot measure out of plane strain; thus arbitrarily rotating the strain field to match the rotations required to align crystal orientation is generally not possible.

When determining whether two grains have undergone equivalent martensitic transformation it is not sufficient to compare a single value, such as the mean strain $(\bar{\varepsilon})$. Averaging transformation strain over the grain surface area obscures fundamental differences in the spatially resolved martensitic transformation behavior. To illustrate this point, Figure 4 shows three grains with similar crystal orientations that have undergone martensitic transformation. Grains $\mathrm{B}$ and $\mathrm{C}$ have similar mean values of strain, but different strain heterogeneity and thus arguably different martensite morphology. Grain B has a uniform distribution of strain, and by inference martensite distribution, while Grain $\mathrm{C}$ has two high strain martensite laths in a low strain austenite matrix. Grains A and B have similar strain ranges (indicative of similar levels of heterogeneity across the grain), but different mean values and overall strain accommodation. In this work, the strain in each grain is characterized by hundreds to thousands of measurement points for a statistically relevant distribution of grain behavior (strain magnitude and heterogeneity).

Grains can contain austenite and martensite delineated by a sharp boundary, as shown in our previous work [46] and in Fig 1d. Grains with sharply delineated martensite lathes tended to show larger ranges of minimum and maximum strain, while grains with higher transformation 
homogeneity showed narrower ranges. This makes sense if one considers each phase and/or variant to produce a narrow range of stains. Under that assumption, when one grain is comprised of one variant, or a homogenous mix of multiple variants with a feature length below the detection limits of SEM-DIC, then a single, tight strain distribution is expected. However, if several regions of a grain each transform to a different variant (or variant mix), these several distributions will sum to a much wider spread across the entire grain.

To remove the effects of data outliers or erroneous points, the limits of the upper and lower quartiles (the middle 50\%) of strain data for each grain were used to define its transformation strain range. While not a direct measure, this strain range is a reasonable way to evaluate transformation homogeneity across a large number of grains. Mean strain values of each grain are not sensitive to these heterogeneities and can thus provide data on the overall degree of transformation in each grain, with higher strains indicating a higher volume fraction of martensite.

\subsection{Comparison of Transformation in Similar Grains}

Here we examine differences in the transformation behavior of similarly oriented grains. Using two metrics - mean strain and strain spread - measured in each grain, it is determined that grains of similar orientation do not transform similarly. The chosen metrics were confirmed to correspond with mean transformation volume fraction and transformation heterogeneity for maps of local transformation strain of a small test group of similarly oriented grains. The sampled population was then expanded to include all those grains with small deviations from low-index orientations. In both populations, no causal link between orientation and extent of martensitic transformation was detected. 
Models based on the phenomenological theory of martensite formation [2,8-15,20] base their macroscopic stress-strain predictions on the assumption that grains with the same orientation, including those related by crystallographic symmetry, produce the same transformation strain in a polycrystalline material. However, in this work it was found that neither grain orientation nor grain size significantly affected either the mean strain or the strain range of individual grains. While averaging single crystal properties across a field of view containing approximately 5000 grains produced roughly correct trends in the macroscopic strain (Figure 3b), predicting transformation from single crystal properties did not produce the correct mean strains inside of each grain and did not capture the wide range of strain they can encompass. Grains can have a large degree of heterogeneity, as seen in Figure 4a, which is not addressed by taking single crystal properties. In addition, predictions using the single variant assumption can deviate significantly from experimental results in both the averaged response of individual grains and the average transformation strain measured across all grains in the sampled microstructure. The experimentally observed mean strains of grains deviated by up to 0.097 from those predicted by assuming transformation of the most favorable variant throughout the grain (see the marked grains in Figure 5). In the field of view shown in Figure 5, the difference between the mean axial strains $\left(\overline{\varepsilon_{\mathrm{xx}}}\right)$ of the predicted and experimental fields was 0.031 . This difference was also observed for other specimens tested, both for specimens machined in the rolling direction and transverse to the rolling direction.

Mean strain and strain range were used to compare the transformation behavior of individual grains. Let us first consider the mean strain. Averaging axial strain over each grain instead of measuring the spatial distribution of transformation — such as the number or spacing of martensite laths - produces a single value for comparison to theoretical predictions. Although it 
is clear that grains do not transform uniformly, but rather can contain a large degree of heterogeneity, this averaging is performed in order to compare experimental results to theoretical predictions that do not account for heterogeneous transformation. The observed transformation heterogeneity in grains, the discrepancy between experimentally measured and theoretically predicted mean strains, and the discrepancy in mean strains between grains of similar orientation are all strong arguments against the validity of assuming transformation similarity based on similar grain orientation. Grains with different mean strain values must have different martensite transformation paths - constituting both the variants and final volume fraction of martensite transformation. In the microstructures examined here, mean grain strain and transformation area fraction exhibited a fairly linear trend (Figure 6), indicating that two grains with similar mean strain values often have similar volume fractions of martensite variants and retained austenite. Points were determined to have transformed to martensite if they achieved $>50 \%$ of the predicted twinned transformation strain-which was taken as an indication they were at least half martensite by a rule of mixtures approach. Volume fractions for each grain were determined by averaging the binary values for each point in the grain. Using this definition, all of the 26 grains with a $49-51 \%$ martensite volume fraction showed a narrow range of grain averaged strain $\left(\overline{\varepsilon_{\mathrm{xx}}}=\right.$ 0.028-0.038). The converse does not hold, however, as the same strain range $\left(\overline{\varepsilon_{\mathrm{xx}}}=0.028-0.038\right)$ contained 258 grains with a mean martensite volume fraction lying at 60\%. Simply considering the mean behaviors of grains is not sufficient to show a general trend in similarity.

We observed that similarly oriented grains can exhibit large variations in mean strain. An example is shown by the two grains marked A and B in Fig. 4a. The bar graphs on top of each grain represent the experimentally measured strain values over that grain surface. As approximately 5.5 million measurements were made over 5300 grains, each grain had nominally 
1000 measurements. The middle $50 \%$ of the strain data is denoted as the thick bar. The $95^{\text {th }}$ percentile bounds are denoted by thin bars. Open circles in the middle of the distribution mark the mean strain of the grain and the median is marked with a crossbar. These grains, despite similar orientations, accommodated significantly different overall strains, with grain A accommodating a much higher mean strain than grain $\mathrm{B}$.

Strain range is indicative of transformation heterogeneity, where a small strain range indicates relatively homogeneous transformation in that area. Grains that fully and uniformly transformed had a narrow strain range, while grains with clearly defined martensite plates and/or untransformed austenite had a larger strain range. The experimentally measured strain ranges of individual grains showed significant variation. Some grains exhibited strain ranges of $+/-0.1 \%$, indicating relatively homogeneous transformation, while in other grains the strain values spanned a range nearly two orders of magnitude larger. These large strain ranges within a single grain are contrary to the phenomenological assumption of complete and homogenous transformation that would result in uniform strain.

The strain range (transformation heterogeneity) of individual grains did not depend on grain orientation or on their mean strain. Similarly oriented grains could exhibit large variations in strain range, as shown by the two grains marked B and C in Figure 4a. These grains had strain ranges of $0.043-0.055$ and $0.033-0.060$ respectively, despite sharing a loading axis of [0.8179 $0.55790 .1404]$ to the fourth significant digit. Grains with similar mean or median strains also did not necessarily have similar strain ranges. For example, again consider Grains B and C in Figure 4. These two grains had nearly identical median strains, but very different ranges of strain values contained within their surface boundaries. A pair of martensite laths cutting through Grain $\mathrm{C}$ as well as an area of untransformed material along the side of this grain account for the wider strain 
range. When averaged, the heterogeneities in Grain $\mathrm{C}$ match the more uniform deformation in Grain B. Considering the width of the strain distribution of each grain reveals differences in the two grains' transformation heterogeneity.

To examine transformation strain and heterogeneity with respect to crystallographic orientation, the full-field strain data at the microstructural length scale was segmented into grainby-grain strain distributions. Each bar in figures 7-10 represents the experimentally-measured surface strain values over grain from the field of view shown in Figure 1d. Similar to figure 4, the middle $50 \%$ of the strain data is denoted as the thick bar, the $95^{\text {th }}$ percentile bounds are denoted by thin bars, the median of each distribution is marked by a crossbar, and open circles mark the mean strain of the grain. The grains were sorted along the x-axis by the misorientation between the [hkl] crystal axis and the sample loading axis. Each grain was color coded by grain size, given as total number of data points contained inside the surface of the grain, showing that there is no apparent effect of size on strain distribution.

Favorable orientations for transformation were examined, including the $\{111\}$ (Figure 7), $\{355\}$ (Figure 8), and to a lesser extent the $\{110\}$ (Figure 9) families of the parent austenite phase. Comparison within and between each of these families shows that the phenomenological assumption that each family of grains has a constant transformation strain does not hold for polycrystals. To determine this, the mean strain and strain range of each grain was normalized by the predicted strain of the austenite-to-twinned-martensite transformation for that grain orientation [20,22], allowing comparison between different orientations. With each of these orientations, there was a wide variation in the mean strain of individual grains in the polycrystal. Although a large portion of the mean strain values fell within $20 \%$ of the predicted orientationdependent axial strains for twinned martensite [20,22], outliers did occur. It is important to note 
that even if these outliers are eliminated, a wide range of transformation strains persists, indicating that final martensite configurations do not solely depend on crystal orientation. Even for grains with distributions within $20 \%$ of the predicted transformation strain, it was possible to find two distributions that shared no overlap between their $95^{\text {th }}$ percentile bounds. Two grains without overlap have distinctly different configurations of martensite in their fully transformed state that lead to completely different strain distributions. Moreover, neither grain size nor misorientation from the preferred crystal axes significantly impacted these values. Finally, these findings were not isolated to orientations favorable for transformation; for example, grains with a $\{100\}$ (Figure 10) orientation along the loading axis also showed significant variation in their mean strains with little dependence on grain size or misorientation.

Nor is it the case that favorably grains exhibited a higher degree of homogeneity. The $\{100\}$, $\{110\},\{111\}$, and $\{355\}$ grain families all showed a similar spread in strain distribution, but exhibited different mean strain values. From the experimentally measured, orientation averaged IPF map in Figure $3 b$, it is determined that the $\{100\}$ grains had a much lower transformation strain when averaged together as compared to the $\{110\}$ and $\{111\}$ grains. However, considering each grain in this family individually in Figures 7 and 9, one can see grains exhibiting transformation strains well above the low collective average. Moreover, the same range of strain spread can be seen in each family of grain orientations. Tracking the strain spread across all orientations (Figure 11a) and grain sizes (Figure 11b), indicates that the heterogeneity does not strongly depend on either orientation or grain size. Despite some trends in mean strain existing across an inverse pole figure (see Figure 3b), plotting the mean strain spread for the same orientations yields the uniform distribution as seen in Figure 11a. Thus, unlike mean strain, the strain range (degree of transformation heterogeneity) did not have a significant dependence on 
grain orientation. The strain range (degree of transformation heterogeneity) of individual grains also did not depend on the grain size. Wide and narrow spreads of strain values were seen in both large and small grains, as shown in Figure 11b and also evident in in Figures 7-10.

\section{Conclusions}

The dependence of grain orientation on stress-induced martensitic transformation in superelastic, polycrystalline Nickel-Titanium sheet was examined at the microstructural length scale. It was found that similarly oriented grains, as determined by the misorientation of the grain relative to the loading direction, do not necessarily transform similarly in either the grain mean strain or strain range. Specifically, grains of similar orientation showed mean strains deviating significantly from predictions of twinned transformation strain and from each other. Grains of similar orientation also showed varying degrees of heterogeneity. Some grains had multiple distinct martensite laths crossing their surface, while others displayed nearly uniform transformation. No dependence was observed between the large variation in mean strain and strain range and the misorientation of the grain from the common crystal axes, nor was any relationship found between this variation and grain size. The findings of this experimental work are in contrast to the assumption in several mean-field theories that similarly oriented grains transform similarly, and in contrast to the assumption that a single preferred variant nucleates and subsumes the entire grain.

\section{Acknowledgements}

This work was supported by the U.S. Department of Energy, Office of Science, Basic Energy Sciences, under Award \#DE-SC0003996. The authors thank Dr. Alan Pelton and Prof. John Shaw for helpful discussions and valuable insight. Portions of this work were performed using 
the FEI Nova Dual Beam FIB at the Electron Microbeam Analysis Lab at the University of Michigan.

\section{References}

[1] Bhattacharya K. Microstructure of Martensite: Why it forms and how it gives rise to the shape memory effect. New York: Oxford University Press Inc.; 2003.

[2] Matsumoto O, Miyazaki S, Otsuka K, Tamura H. Crystallography of martensitic transformation in Ti-Ni single crystals. Acta Metall 1987;35:2137-44.

[3] Knowles K, Smith D. The crystallography of the martensitic transformation in equiatomic NickelTitanium. Acta Metall 1980;29:101-10.

[4] Hane K, Shield T. Microstructure in the cubic to monoclinic transition in titanium-nickel shape memory alloys. Acta Mater 1999;47:2603-17.

[5] Miyazaki S, Otsuka K, Wayman C. Morphological changes associated with the R-phase and martensitic Ti-Ni single crystals. ISIJ Int 1989;29:423-9.

[6] Gall K, Sehitoglu H, Chumlyakov Y, Kireeva I. Tension-compression asymmetry of the stressstrain response in aged single crystal and polycrystalline NiTi. Acta Mater 1999;47:1203-17.

[7] Miyazaki S, Kimura S, Takei F, Miura T, Otsuka K, Suzuki Y. Shape memory effect and pseudoelasticity in a Ti-Ni single crystal. Scr Metall 1983;17:1057-62.

[8] Savi M, Paiva A, Baeta-Neves A, Pacheco P. Phenomenological modeling and numerical simulation of shape memory alloys: A thermo-plastic-phase transformation coupled model. J Intell Mater Syst Struct 2002;13:261-73.

[9] Ren W, Li H, Song G. Phenomenological modeling of the cyclic behavior of superelastic shape memory alloys. Smart Mater Struct 2007;16:1083-9.

[10] Arghavani J, Auricchio F, Naghdabadi R, Reali A, Sohrabpour S. A 3-D phenomenological constitutive model for shape memory alloys under multiaxial loadings. Int J Plast 2010;26:976-91. 
[11] Bouvet C, Calloch S, Lexcellent C. A phenomenological model for pseudoelasticity of shape memory alloys under multiaxial proportional and nonproportional loadings. Eur J Mech - A/Solids 2004;23:37-61.

[12] Thamburaja P. A finite-deformation-based phenomenological theory for shape-memory alloys. Int J Plast 2010;26:1195-219.

[13] Patoor E, Lagoudas D, Entchev P, Brinson L, Gao X. Shape memory alloys, Part I: General properties and modeling of single crystals. Mech Mater 2006;38:391-429.

[14] Lagoudas D, Entchev P, Popov P, Patoor E, Brinson L, Gao X. Shape memory alloys, Part II: Modeling of polycrystals. Mech Mater 2006;38:430-62.

[15] Lagoudas D, Hartl D, Chemisky Y, Machado L, Popov P. Constitutive model for the numerical analysis of phase transformation in polycrystalline shape memory alloys. Int J Plast 2012;32$33: 155-83$.

[16] Richards A, Lebensohn R, Bhattacharya K. Interplay of martensitic phase transformation and plastic slip in polycrystals. Acta Mater 2013;61:4384-97.

[17] Miyazaki S, Kimura S, Otsuka K, Suzuki Y. The habit plane and transformation strains associated with the martensitic transformation in Ti-Ni single crystals. Scr Metall 1984;18:883-8.

[18] Sehitoglu H, Karaman I, Anderson R, Zhang X, Gall K, Maier H, et al. Compressive response of NiTi single crystals. Acta Mater 2000;48:3311-26.

[19] Gall K, Dunn M, Liu Y, Labossiere P, Sehitoglu H, Chumlyakov Y. Micro and macro deformation of single crystal NiTi. J Eng Mater Technol 2002;124:238.

[20] Buchheit T, Kumpf S, Wert J. Modeling the stress-induced transformation behavior of shape memory alloy single crystals. Acta Metall Mater 1995;43:4189-99.

[21] Falk F. Pseudoelastic stress-strain curves of polycrystalline shape memory alloys calculated from single crystal data. Int J Eng Sci 1989;21.

[22] Buchheit T, Wert J. Modeling the effects of stress state and crystal orientation on the stressinduced transformation of NiTi single crystals. Metall Mater Trans A 1994;25:2383-9. 
[23] Norfleet D, Sarosi P, Manchiraju S, Wagner M, Uchic M, Anderson P, et al. Transformationinduced plasticity during pseudoelastic deformation in Ni-Ti microcrystals. Acta Mater 2009;57:3549-61.

[24] Manjeri R, Qiu S, Mara N, Misra A, Vaidyanathan R. Superelastic response of [111] and [101] oriented NiTi micropillars. J Appl Phys 2010;108:023501.

[25] Frick C, Orso S, Arzt E. Loss of pseudoelasticity in nickel-titanium sub-micron compression pillars. Acta Mater 2007;55:3845-55.

[26] Frick C, Lang T, Spark K, Gall K. Stress-induced martensitic transformations and shape memory at nanometer scales. Proc. Int. Conf. Shape Mem. Superelastic Technol., 2006, p. 99-111.

[27] Frick C, Clark B, Orso S, Sonnweber-Ribic P, Arzt E. Orientation-independent pseudoelasticity in small-scale NiTi compression pillars. Scr Mater 2008;59:7-10.

[28] Miyazaki S, No V, Kitamura K, Khantachawana A, Hosoda H. Texture of Ti-Ni rolled thin plates and sputter-deposited thin films. Int J Plast 2000;16:4-8.

[29] Yuan W, Yi S. Pseudo-elastic strain estimation of textured TiNi shape memory alloys. Mater Sci Eng A 1999;271:439-48.

[30] Inoue H, Miwa N, Inakazu N. Texture and shape memory strain in TiNi alloy sheets. Acta Mater $1996 ; 44: 4825-34$.

[31] Shu Y, Bhattacharya K. The influence of texture on the shape-memory effect in polycrystals. Acta Mater 1998;46:5457-73.

[32] Miyazaki S, Ishida A. Martensitic transformation and shape memory behavior in sputter-deposited TiNi-base thin films. Mater Sci Eng A 1999;273-275:106-33.

[33] Bhattacharya K, Kohn RV. Symmetry, texture and the recoverable strain of shape-memory polycrystals. Acta Mater 1996;44:529-42.

[34] Mao S, Luo J, Zhang Z, Wu M, Liu Y, Han X. EBSD studies of the stress-induced B2-B19' martensitic transformation in NiTi tubes under uniaxial tension and compression. Acta Mater 2010;58:3357-66. 
[35] Mehta A, Gong X, Imbeni V, Pelton A, Ritchie R. Understanding the deformation and fracture of nitinol endovascular stents using in situ synchrotron X-ray microdiffraction. Adv Mater 2007;19:1183-6.

[36] Brinson L. Stress-induced transformation behavior of a polycrystalline NiTi shape memory alloy: micro and macromechanical investigations via in situ optical microscopy. J Mech Phys Solids 2004;52:1549-71.

[37] Kammers A, Daly S. Digital image correlation under scanning electron microscopy: Methodology and validation. Exp Mech 2013;53:1743-61.

[38] Sutton M, Li N, Garcia D, Cornille N, Orteu J-J, McNeill S, et al. Scanning electron microscopy for quantitative small and large deformation measurements Part II: Experimental validation for magnifications from 200 to 10,000. Exp Mech 2007;47:789-804.

[39] Sutton M, Li N, Joy D, Reynolds A, Li X. Scanning electron microscopy for quantitative small and large deformation measurements Part I: SEM imaging at magnifications from 200 to 10,000. Exp Mech 2007;47:775-87.

[40] Schreier H, Orteu J-J, Sutton M. Image Correlation for Shape, Motion and Deformation Measurements. Boston, MA: Springer US; 2009.

[41] Correlated Solutions. VIC-2D 2009.

[42] Pelton A. Personal Communication 2013.

[43] Pelton A, Dicello J, Miyazaki S. Optimization of processing and properties of medical-grade nitinol wire. Minim Invasive Ther Allied Technol 2000;9:107-18.

[44] Kammers A, Daly S. Self-assembled nanoparticle surface patterning for improved digital image correlation in a scanning electron microscope. Exp Mech 2013;53:1333-41.

[45] Turner T, Shade P, Schuren J, Groeber M. The influence of microstructure on surface strain distributions in a nickel micro-tension specimen. Model Simul Mater Sci Eng 2013;21:015002.

[46] Kimiecik M, Jones J, Daly S. Quantitative studies of microstructural phase transformation in Nickel-Titanium. Mater Lett 2013;95:25-9. 


\section{Figure Captions}

Figure 1: (a) Sample dimensions; (b) EBSD scan of the loading direction orientation used to determine grain boundaries and local orientation; (c) area fraction vs. grain diameter; (d) grain boundaries aligned with the nanoparticle DIC pattern; (e) grain boundaries displayed as white lines in the strain field to correlate strain and orientation fields for analysis. The black spots in (b) and (e) are the same platinum markers shown in (d) and are used to align the data sets and delineate a nominal $100 \mu \mathrm{m}$ square.

Figure 2: Schematic showing the isomorphism of two grains with similar loading direction orientations. Any two grains with similar loading axes can have their orientation mapped to one another by a rigid rotation about the loading axis, which has no effect on the measured loading axis strain.

Figure 3: (a) Inverse pole figure (IPF) map of accommodated strain from martensite transformation based on loading direction of single crystals from [10]; and (b) polycrystalline SEM-DIC data from these experiments. The overall trends of low strains along $\{100\}$ directions and highest strains along $\{111\}$ directions remain intact. However, the measured polycrystalline strain shows $\sim 3 \%$ less strain over much of the IPF space.

Figure 4: (a) Selection of strain distributions from three grains with similar orientations as shown in (b). The thick line represents the middle $50 \%$ of data and the thin line constitutes 95 th percentile bounds for each grain. The median of each grain's strain distribution is marked with a crossbar while the mean is shown as an open circle. A and B show the same level of 
homogeneity (strain spread) despite different mean/median strain levels, while B and C show the same median strain with $\mathrm{C}$ showing much larger heterogeneity of transformation.

Figure 5: (a) Predicted transformation strain from phenomenological theory; (b) experimentallymeasured strains; and (c) the difference between (a) and (b). Grains with the highest deviation between predicted and measured transformation strain are circled in each field. Summaries of the strain fields are given in Table 1.

Figure 6: Mean axial strain in the grain as a function of martensite area fraction. Points were partitioned into austenite or martensite using a rule of mixtures approach. Twinned martensite strain in each grain was calculated; if the measured axial strain at a point was closer to the twinned strain than the nominal elastic strain $\left(\varepsilon_{\mathrm{xx}}=0.01\right)$, the point was considered to be fully martensite. Binary states were then averaged to determine the martensite area fraction of each grain.

Figures 7-10: Individual grain strain distributions for grains oriented along different low-index crystallographic directions. To facilitate comparison between the different orientations, all strain values were normalized to the predicted transformation strain of twinned martensite, which is dependent on the crystal orientation. To limit clutter in this image, grains with a diameter of less than $0.5 \mu \mathrm{m}$ were omitted.

Figure 11: Strain spread of grains is not controlled by (a) loading axis orientation or (b) grain size. The same mean strain spread can be seen for most orientations in (a) with no trends across 
the orientation space. Similarly, comparing the grain size and strain spread of each gran in (b) yields no trend beyond an increasing lower bound in strain spread for larger grains. However, the same strain spreads observed in the largest grains can be found in a large number of smaller grains as well. Additionally, it is unclear if the lower bound is a true trend or a consequence of the larger number of sample points in the larger grains.

Table 1: Summary of full field strain maps from Figure 5. 


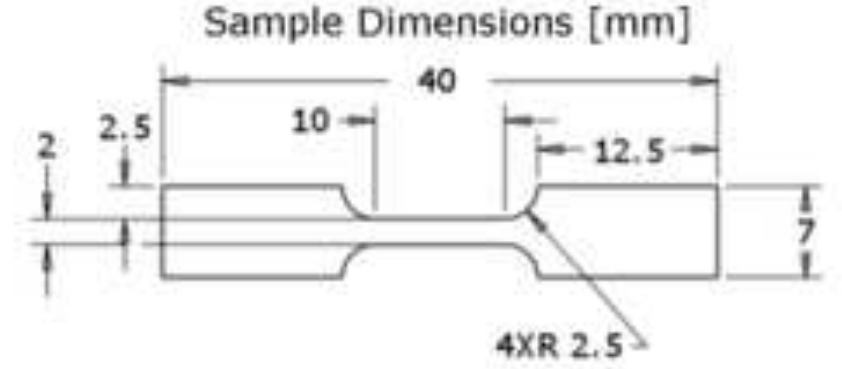

(a)

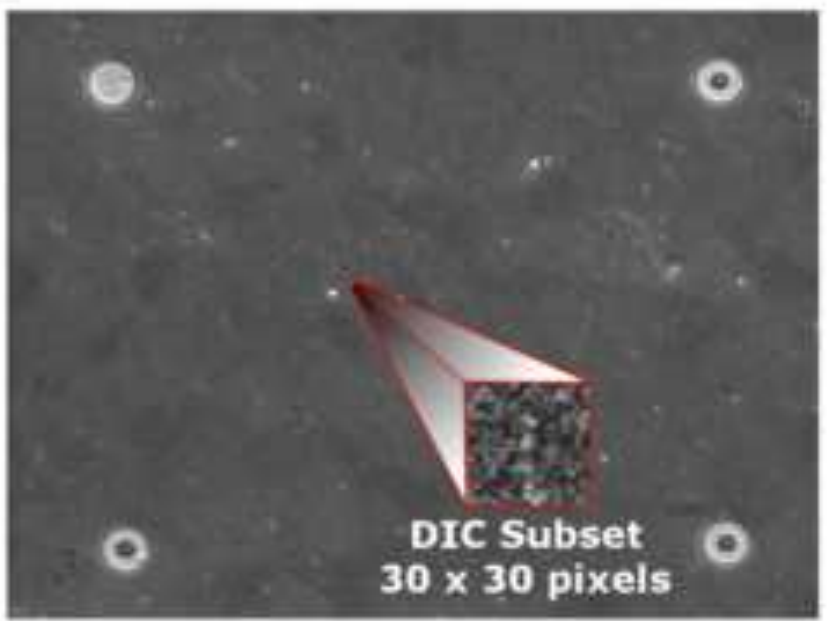

(d)

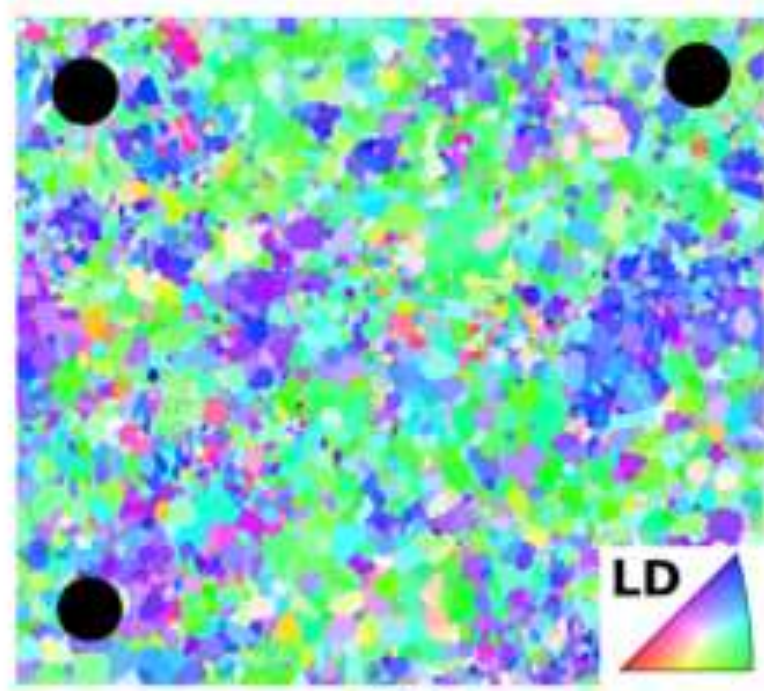

(b)

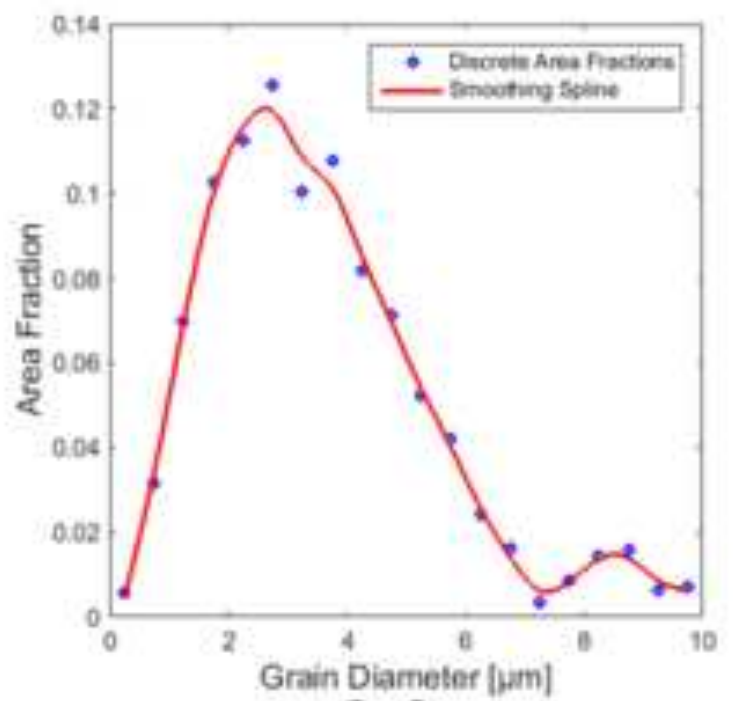

(c)

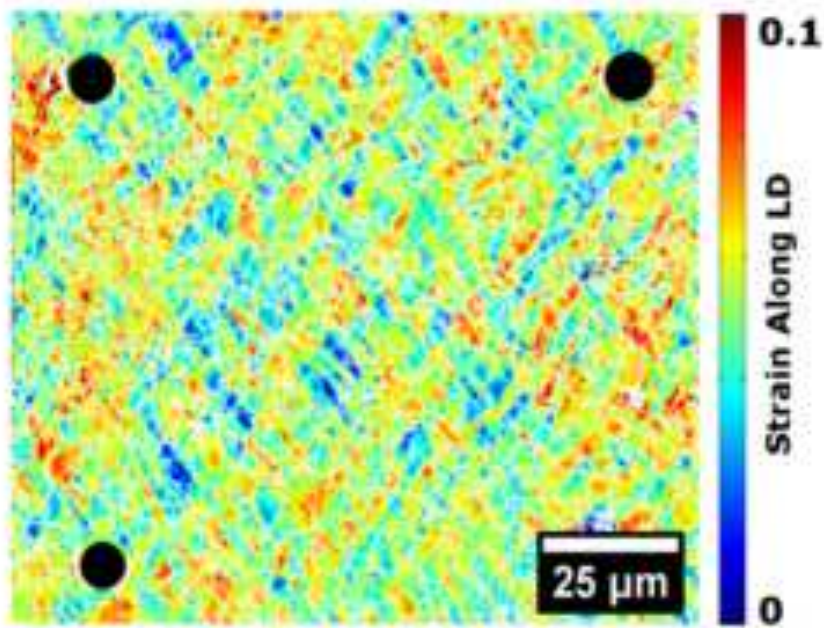

(e) 


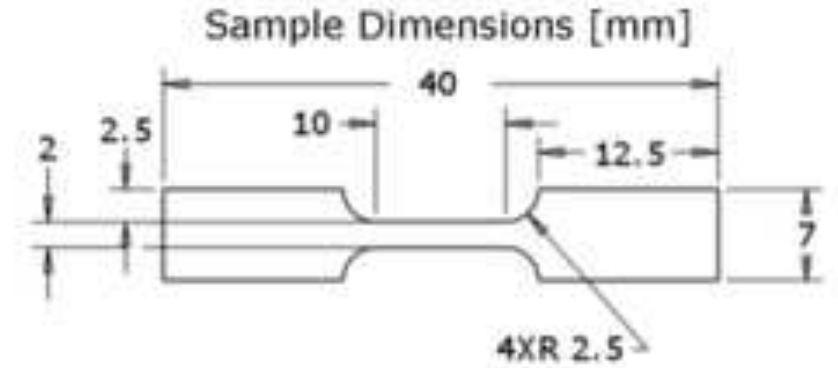

(a)

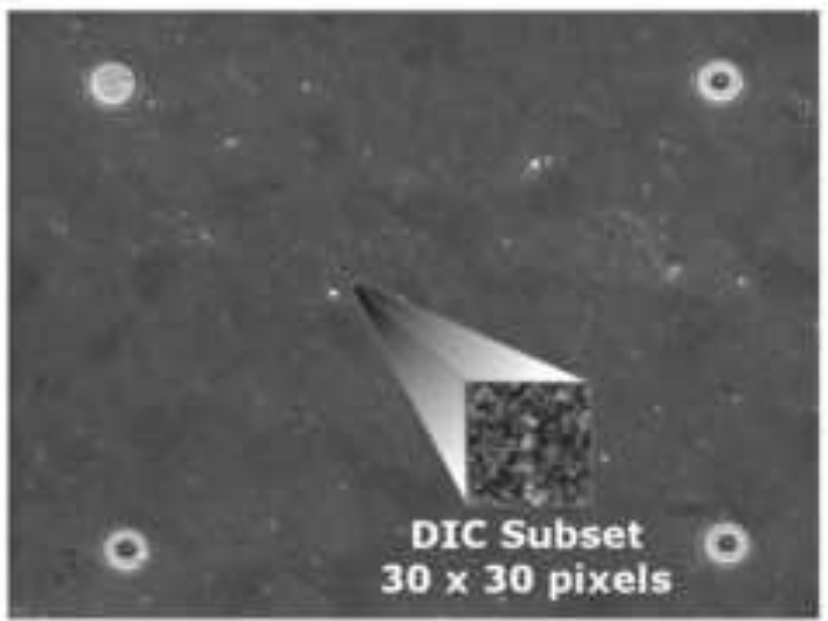

(d)

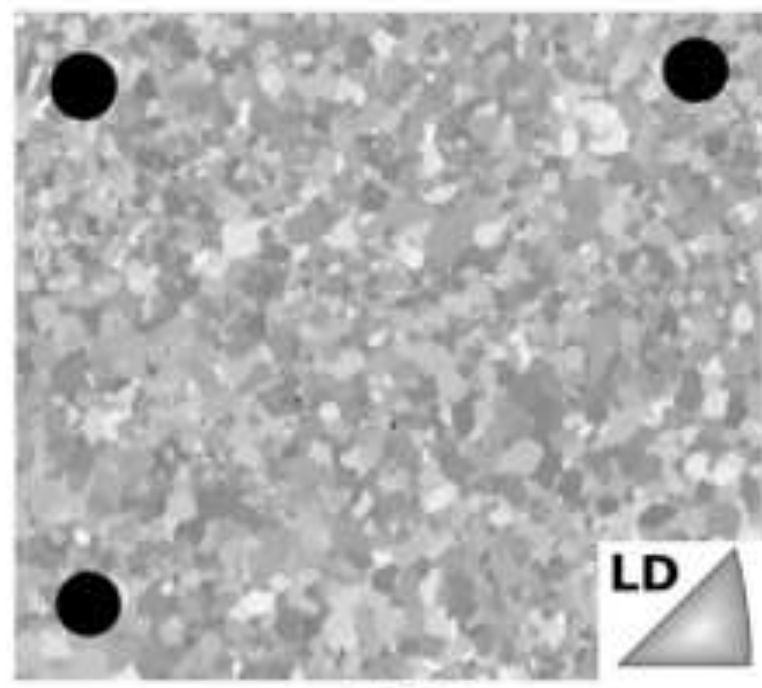

(b)

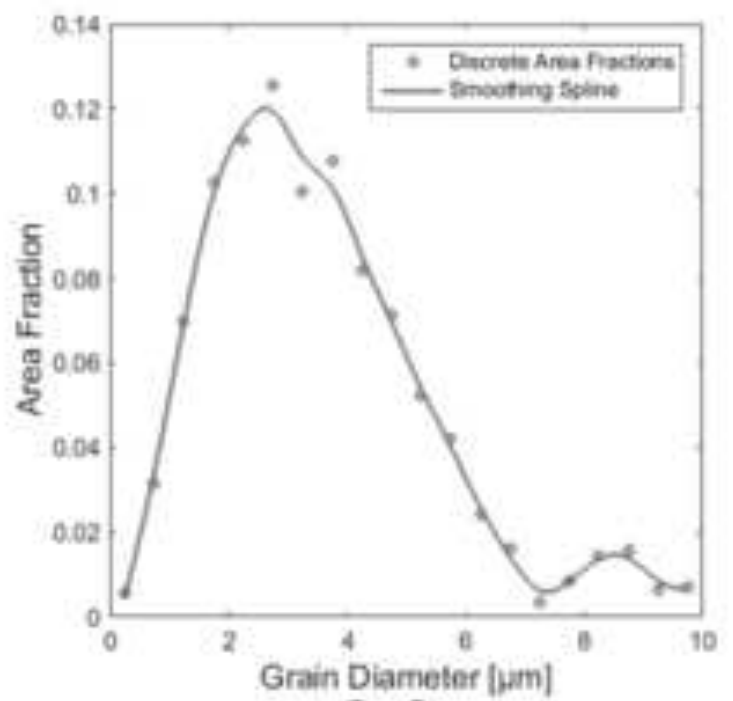

(c)

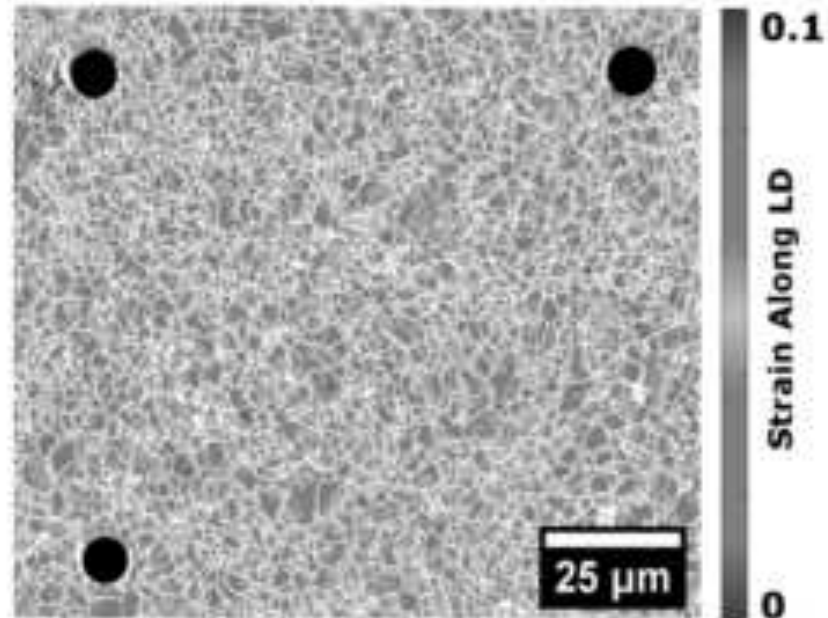

(e) 

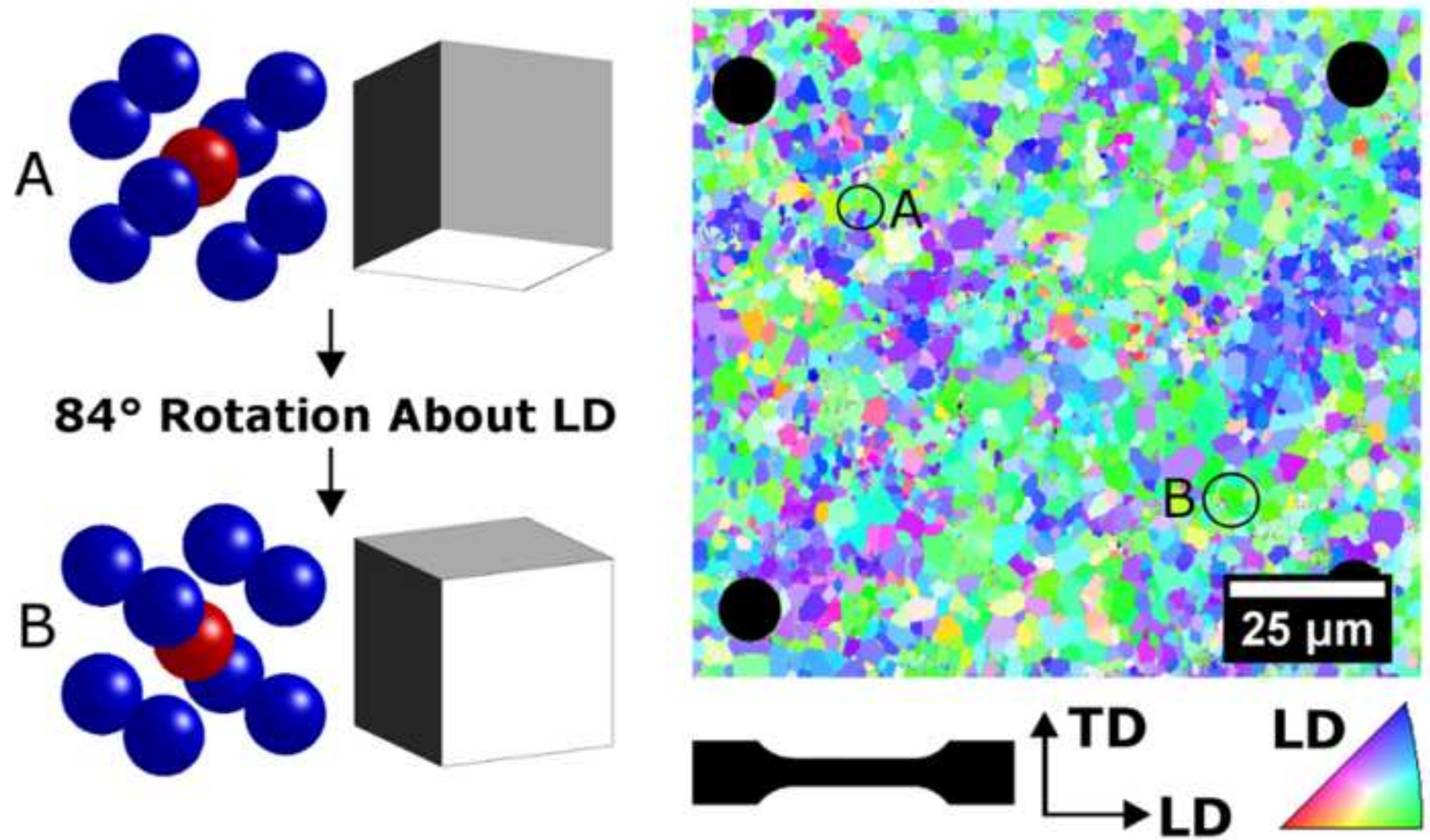

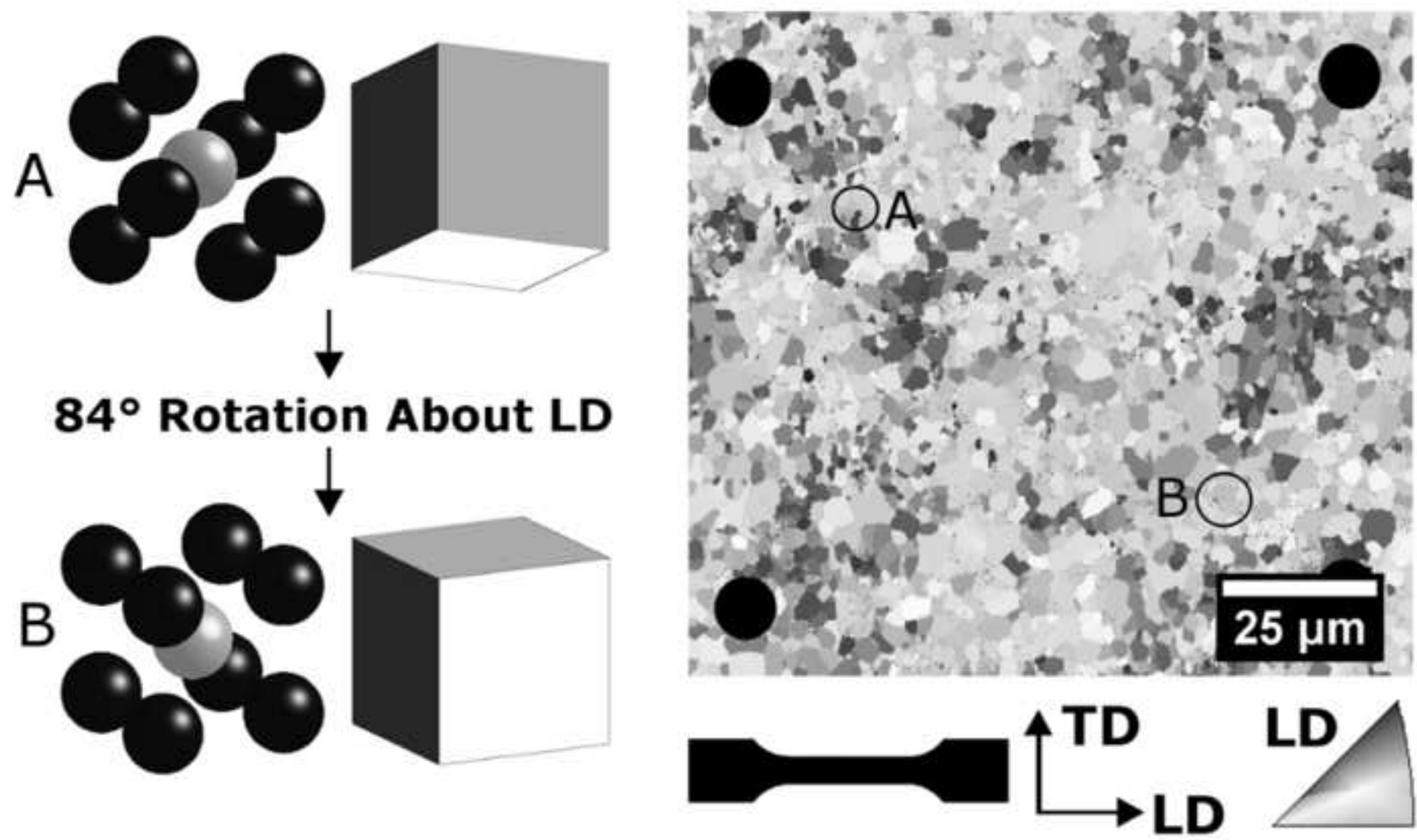


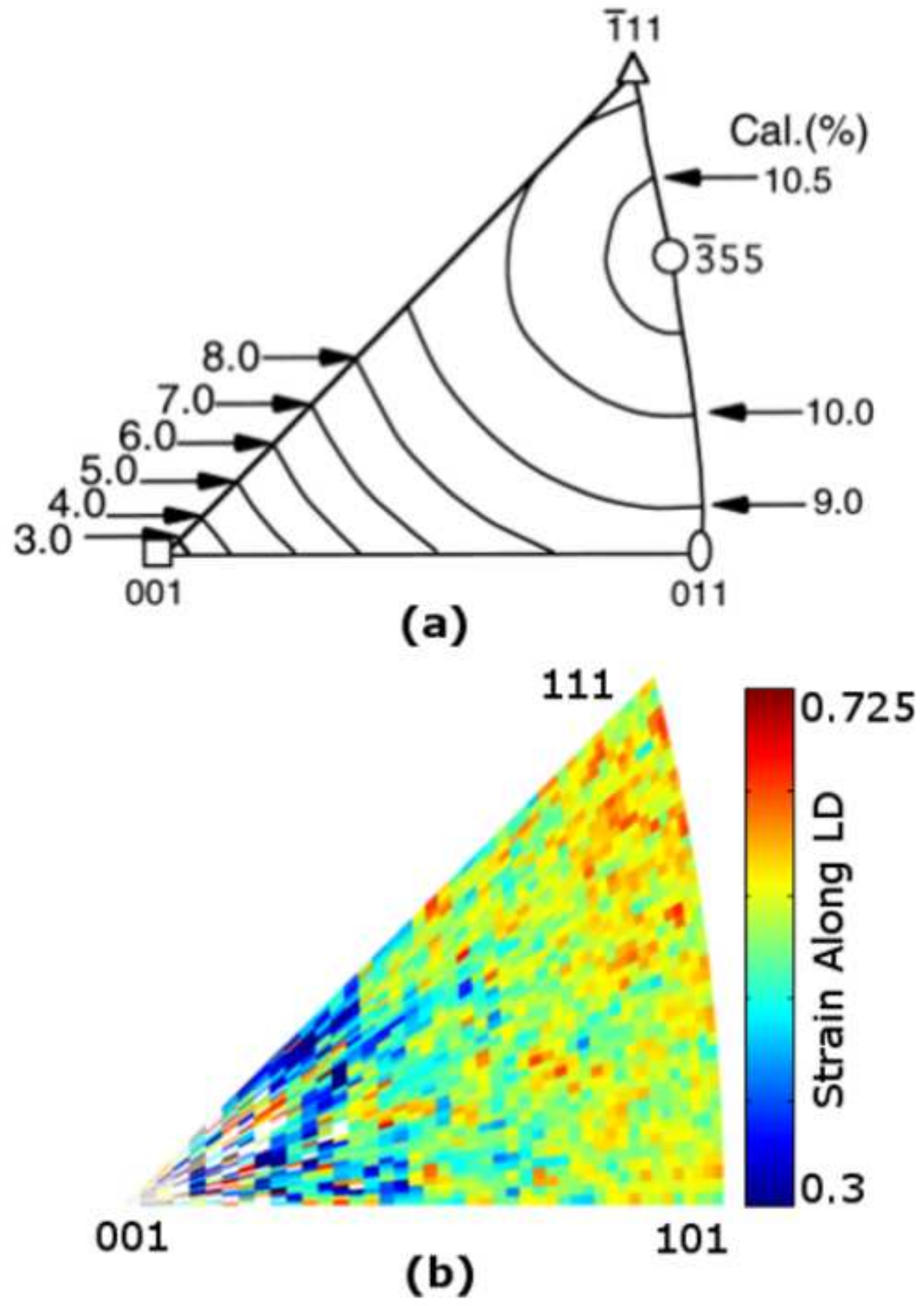




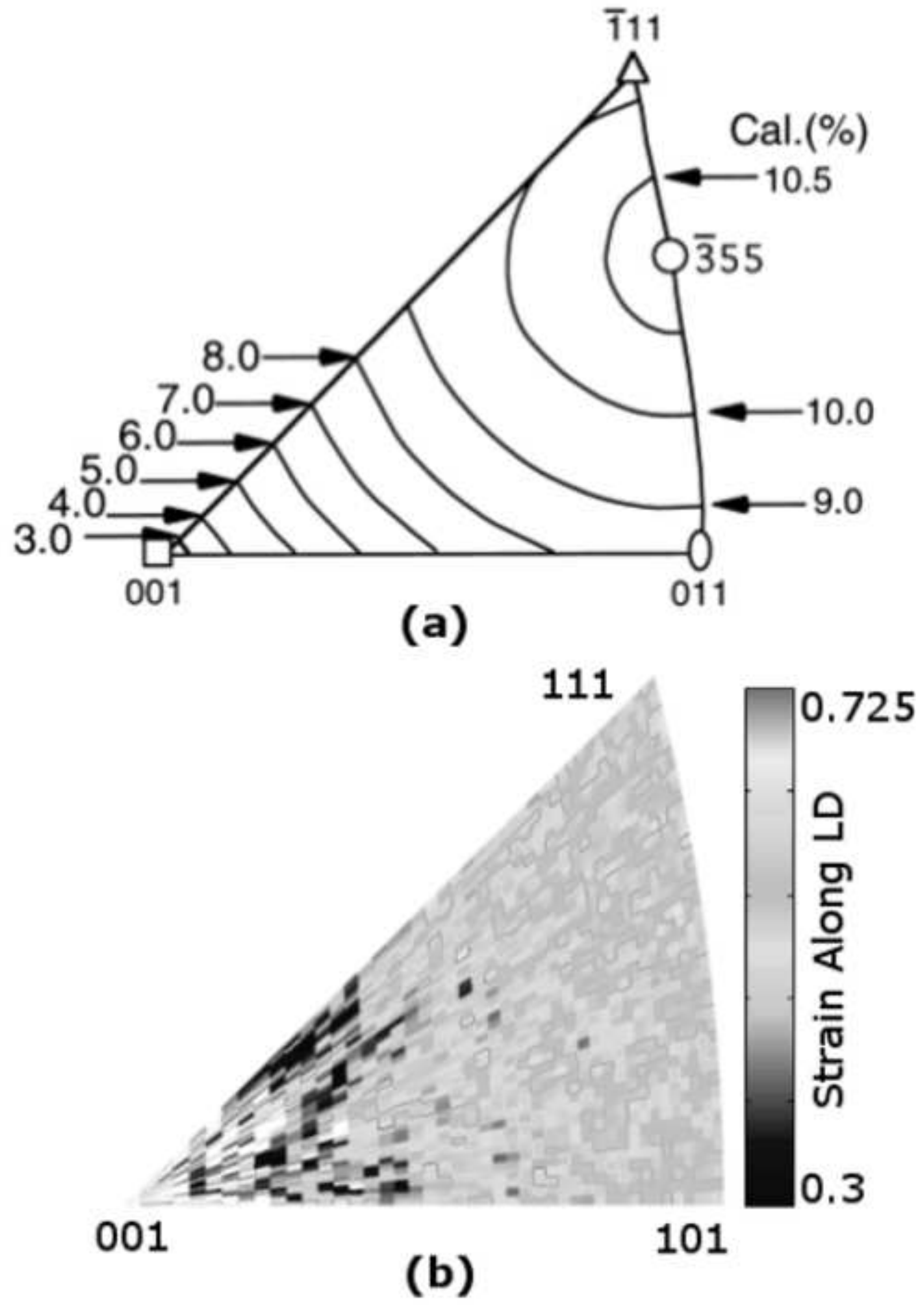



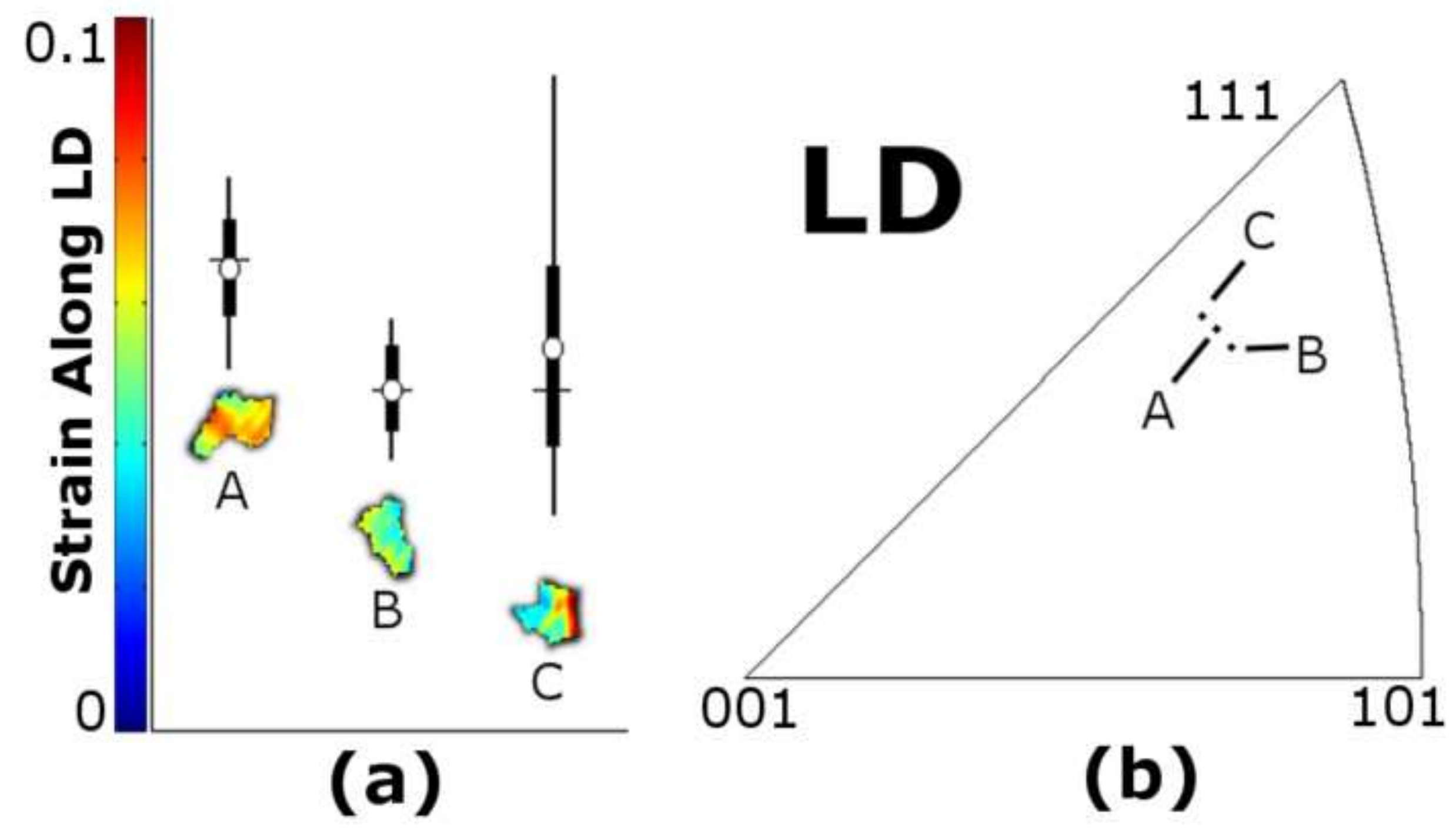

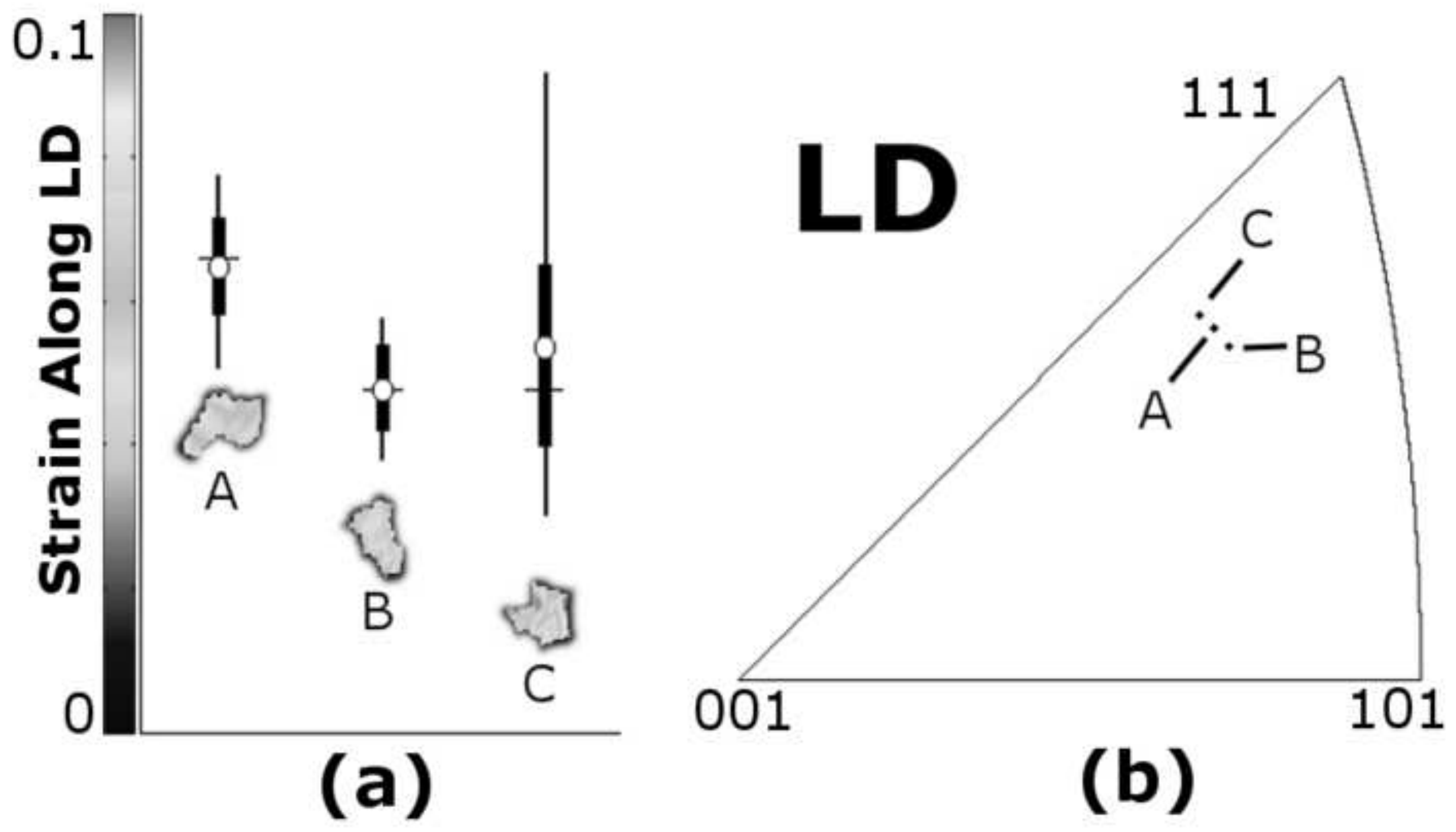


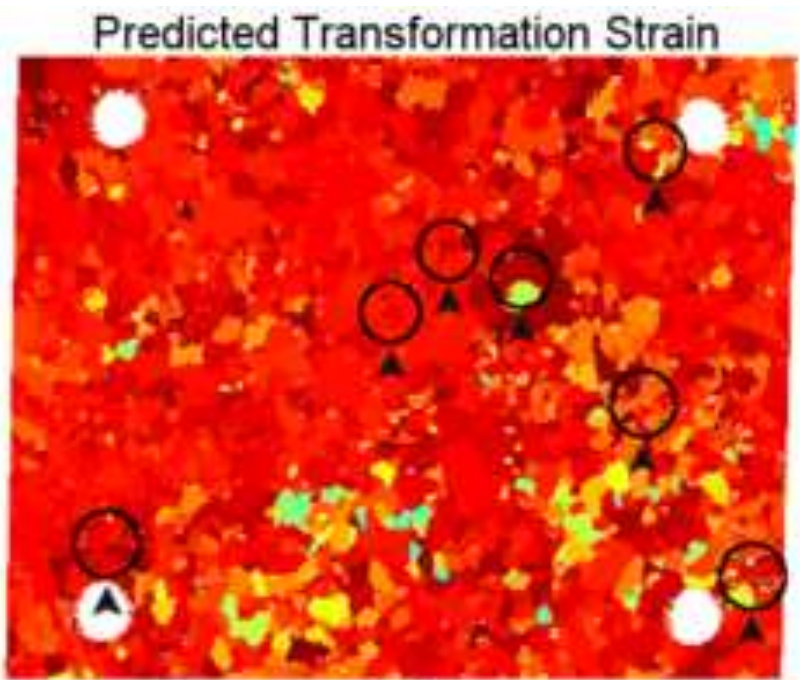

(a)

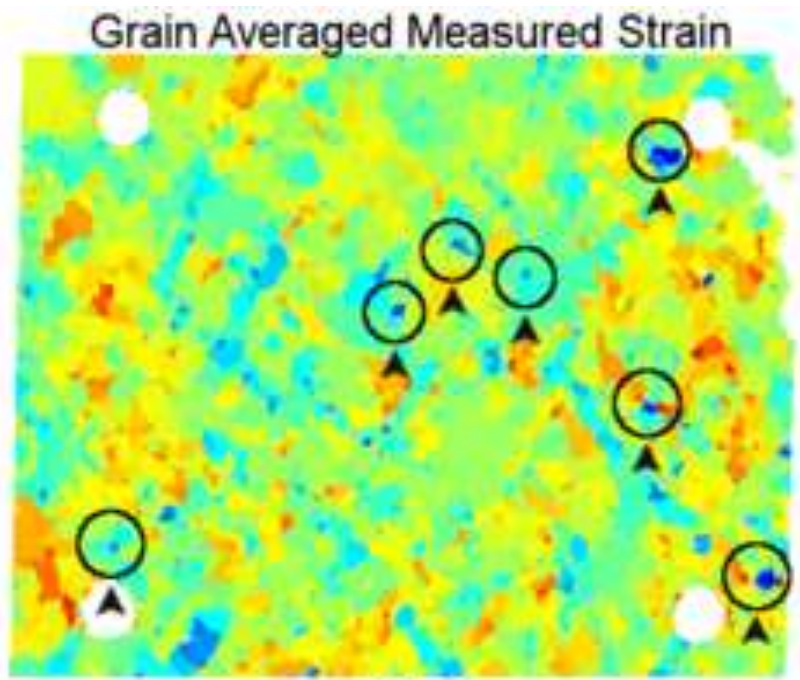

(b)

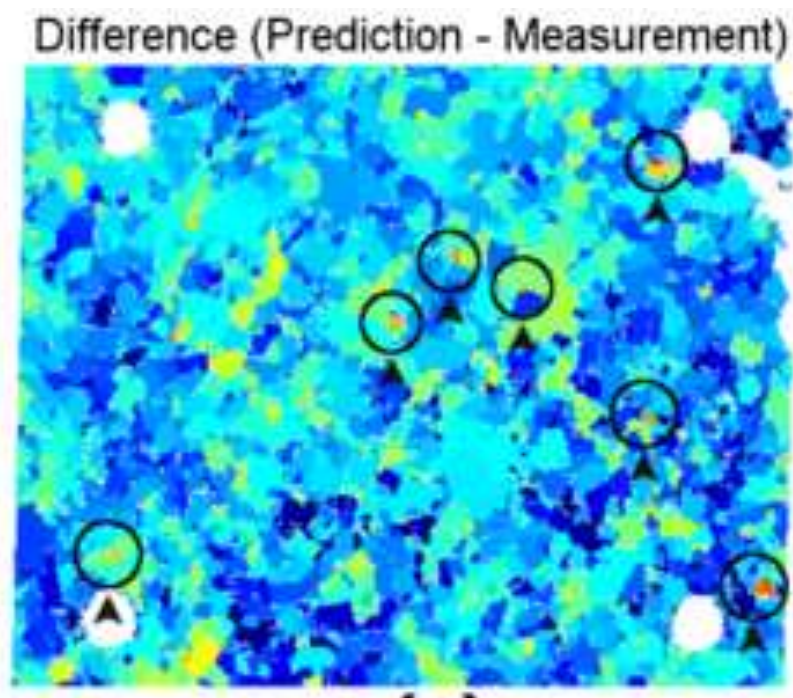

(c)

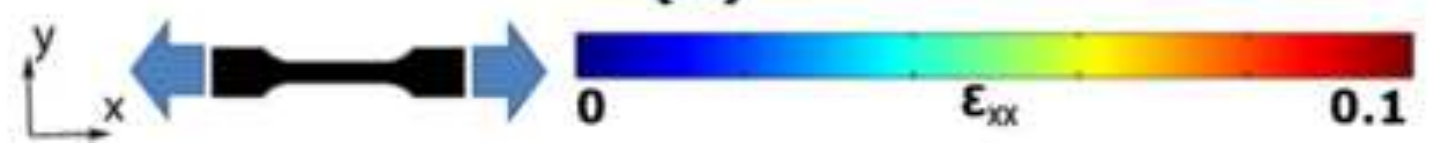




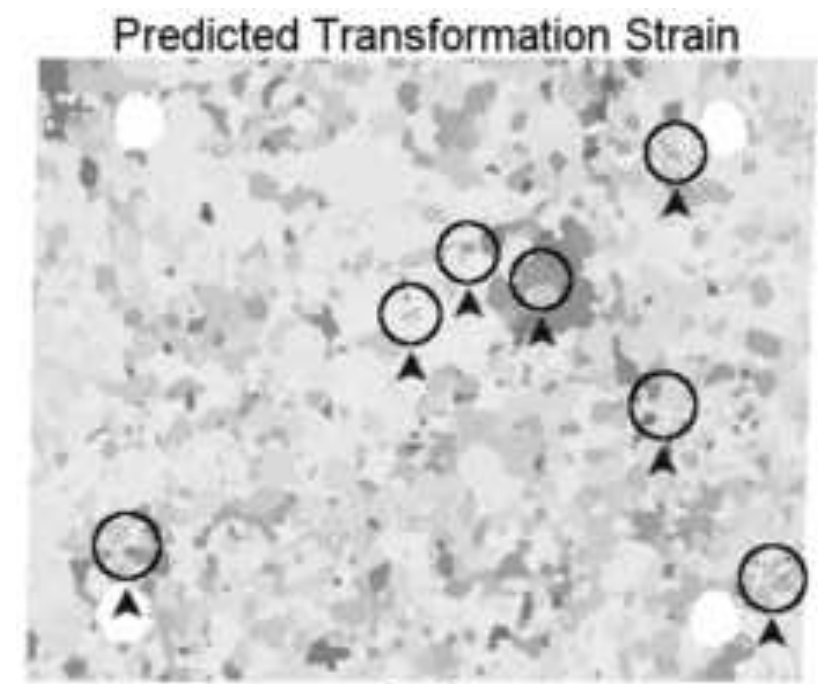

(a)

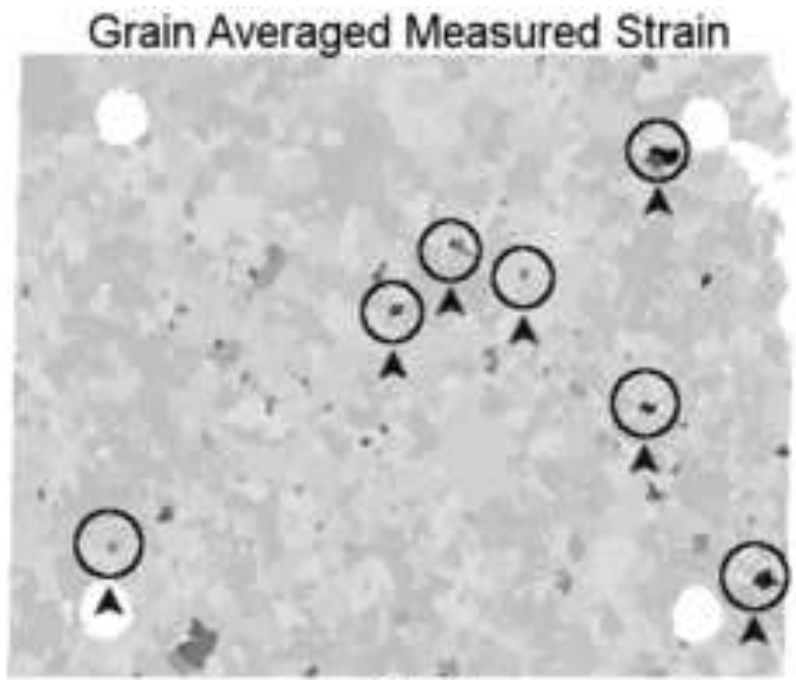

(b)
Difference (Prediction - Measurement)
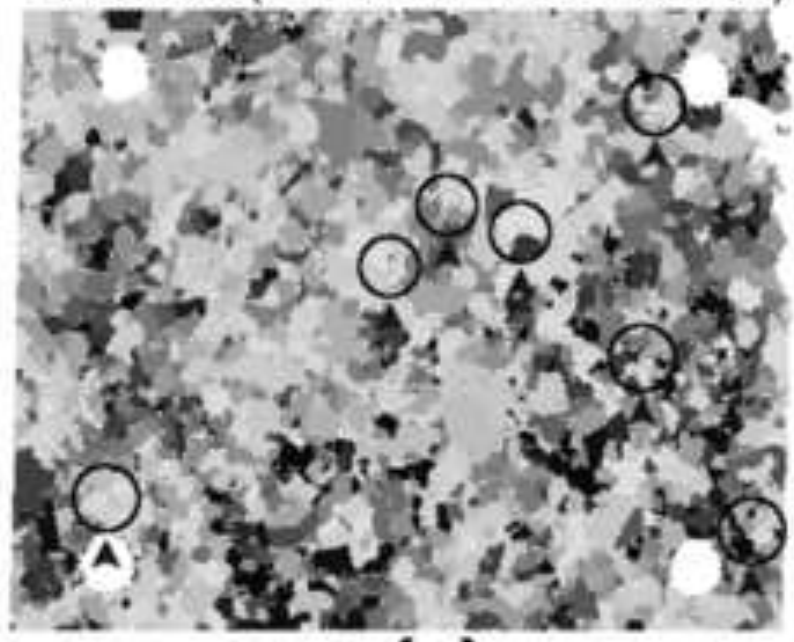

(c)

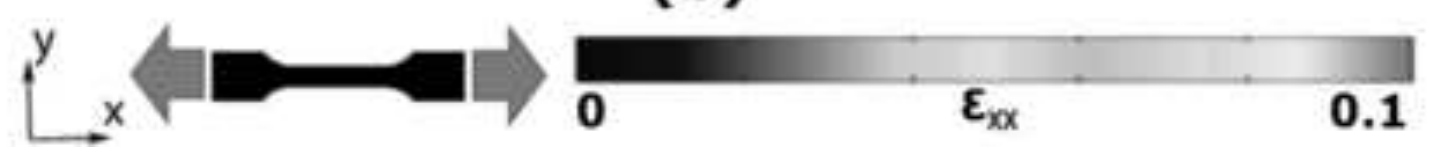




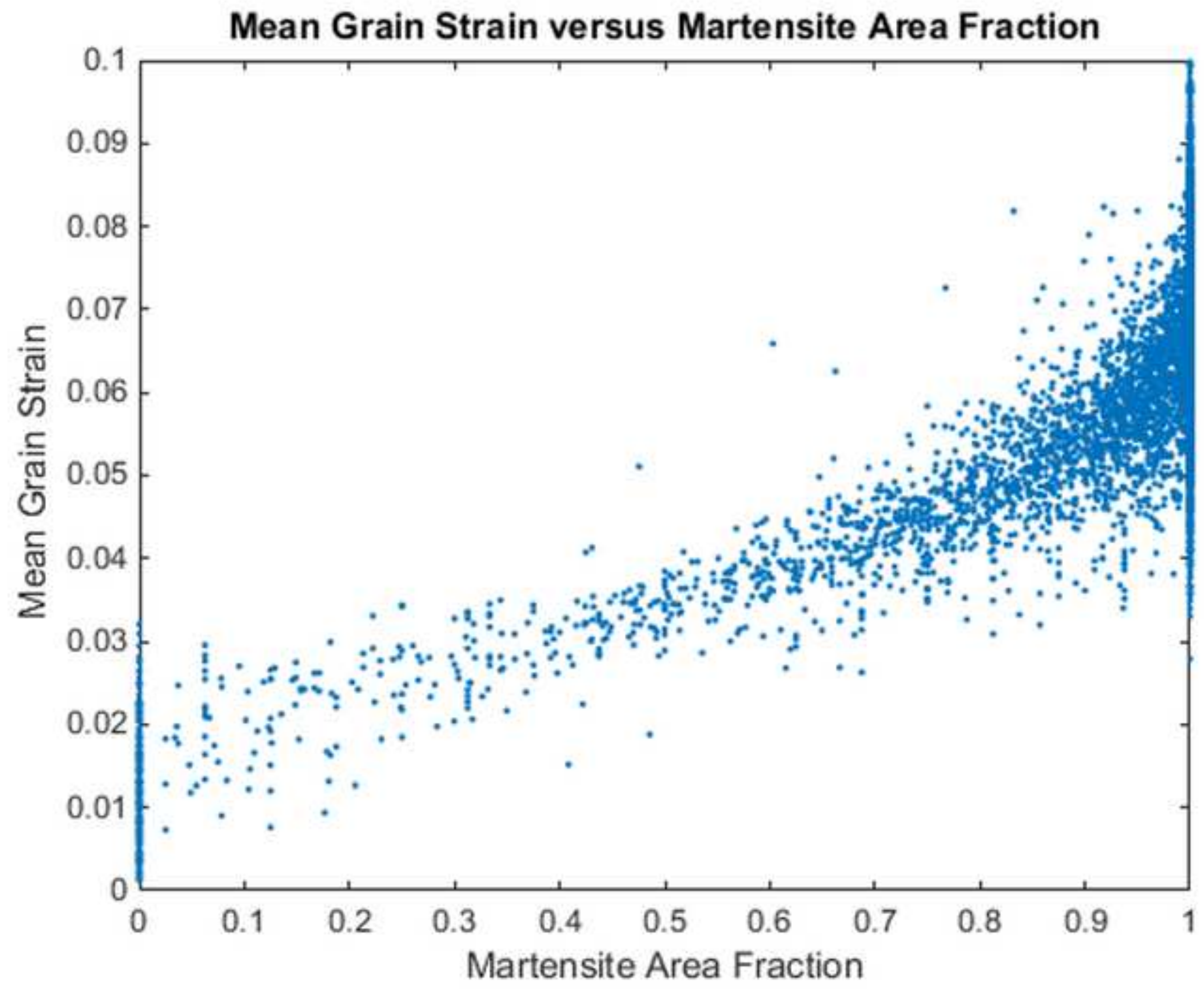




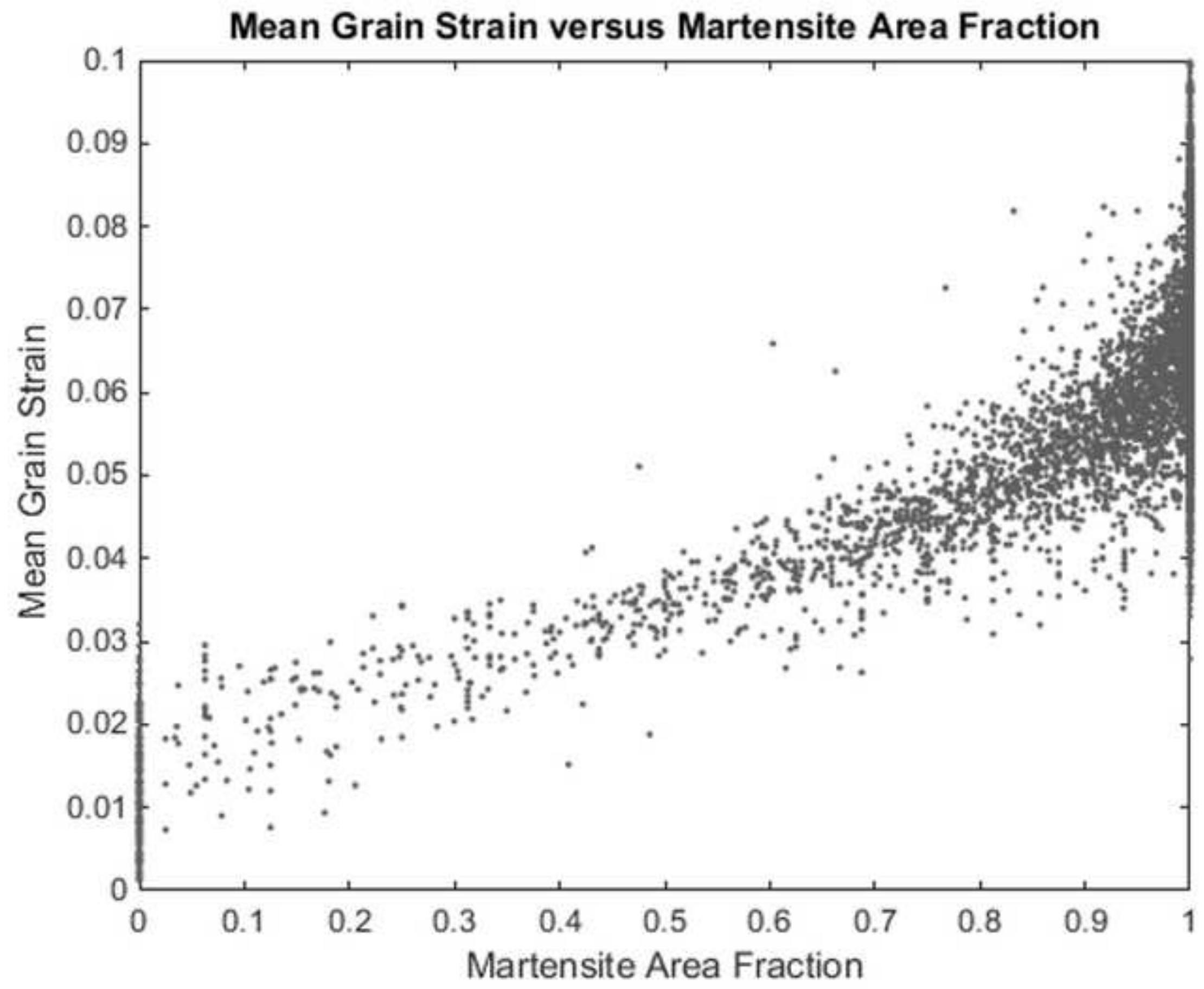




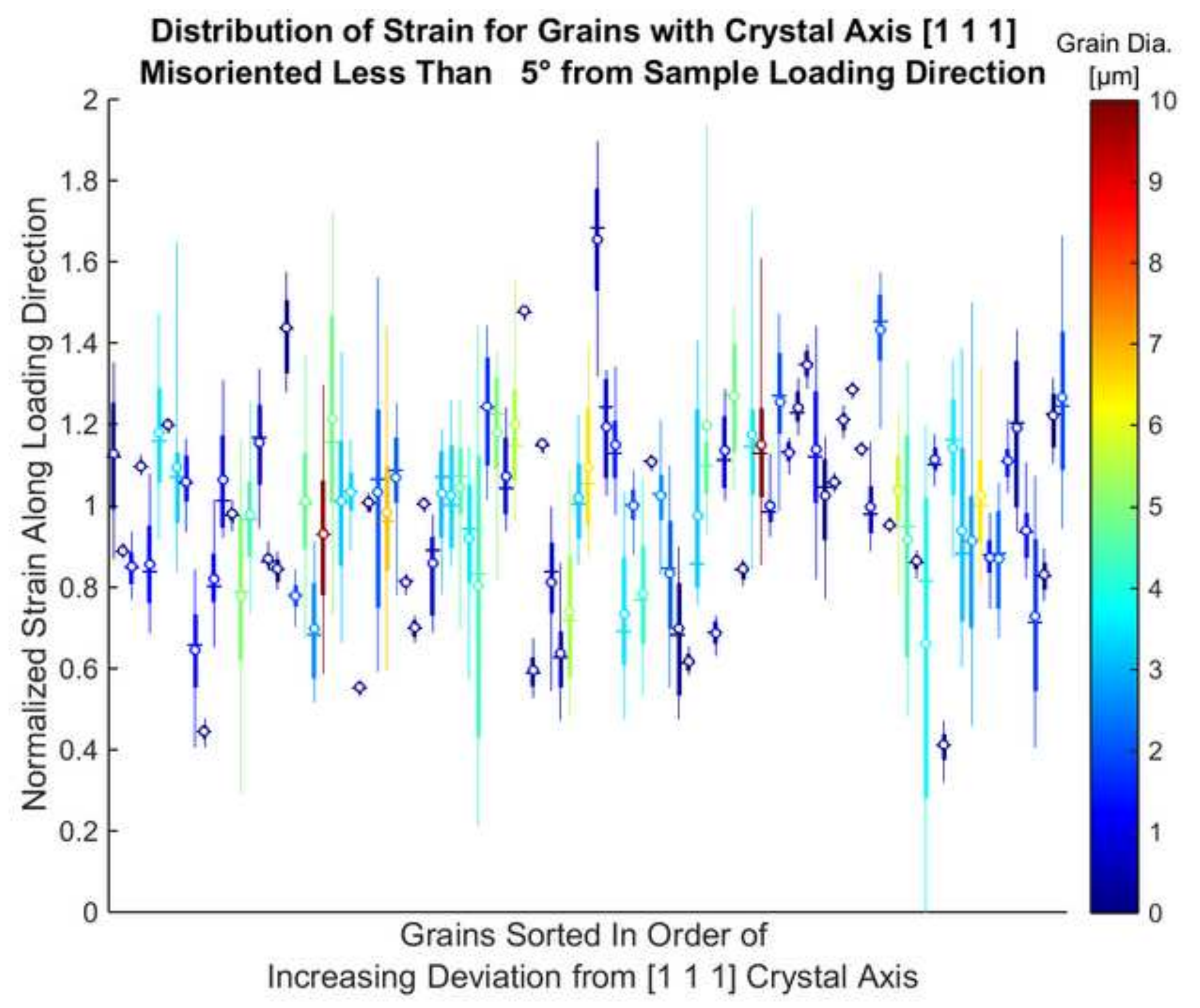




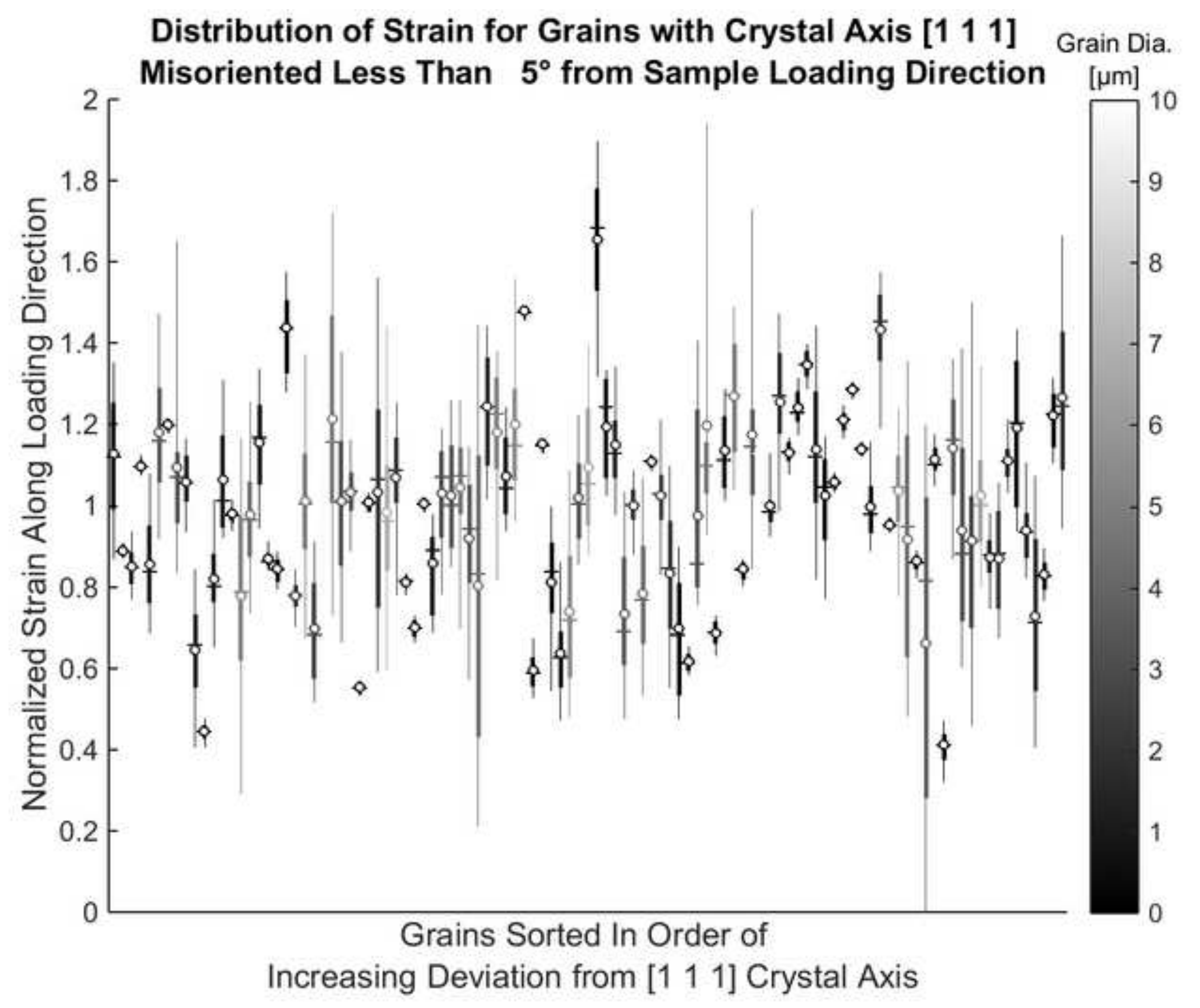




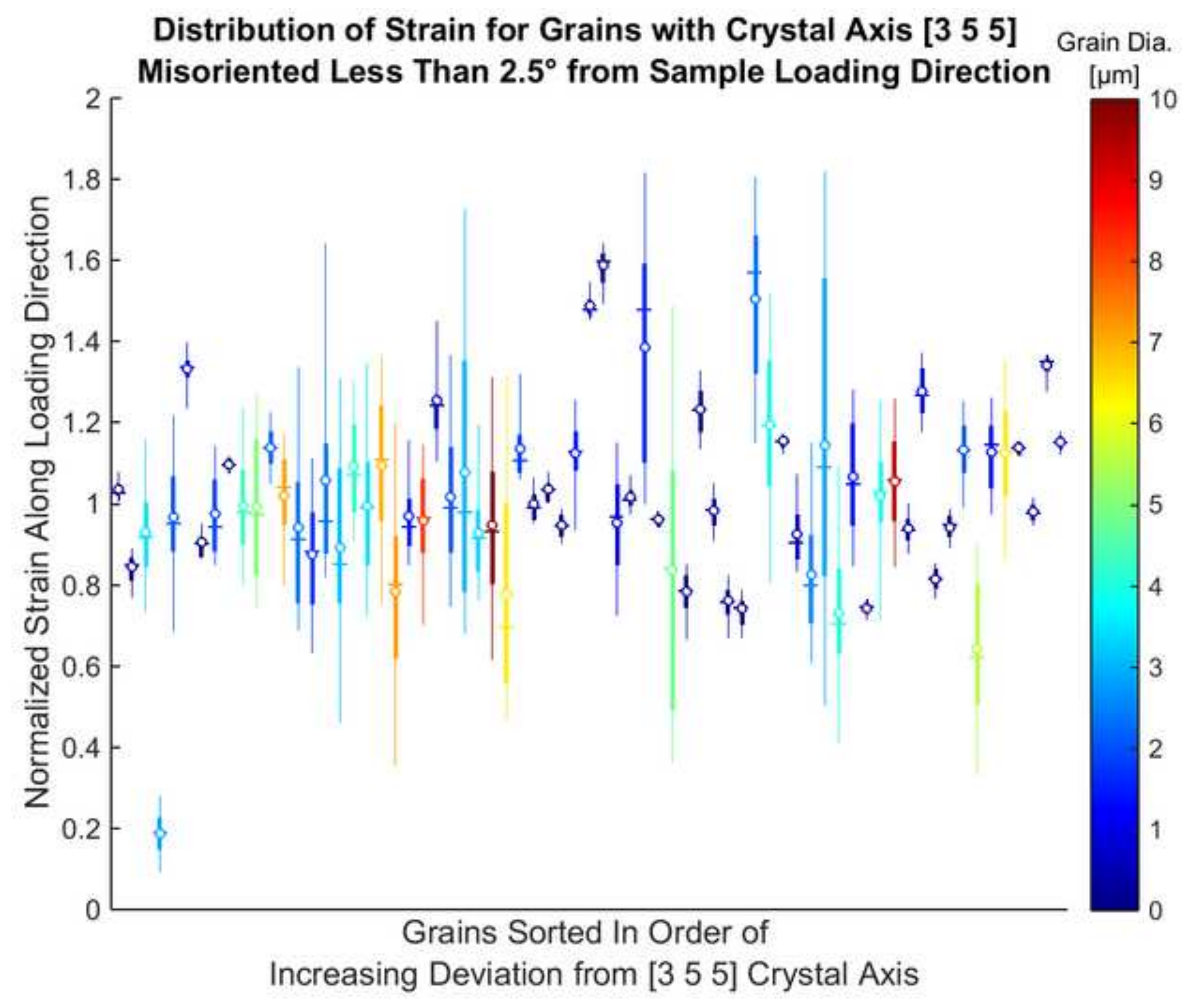




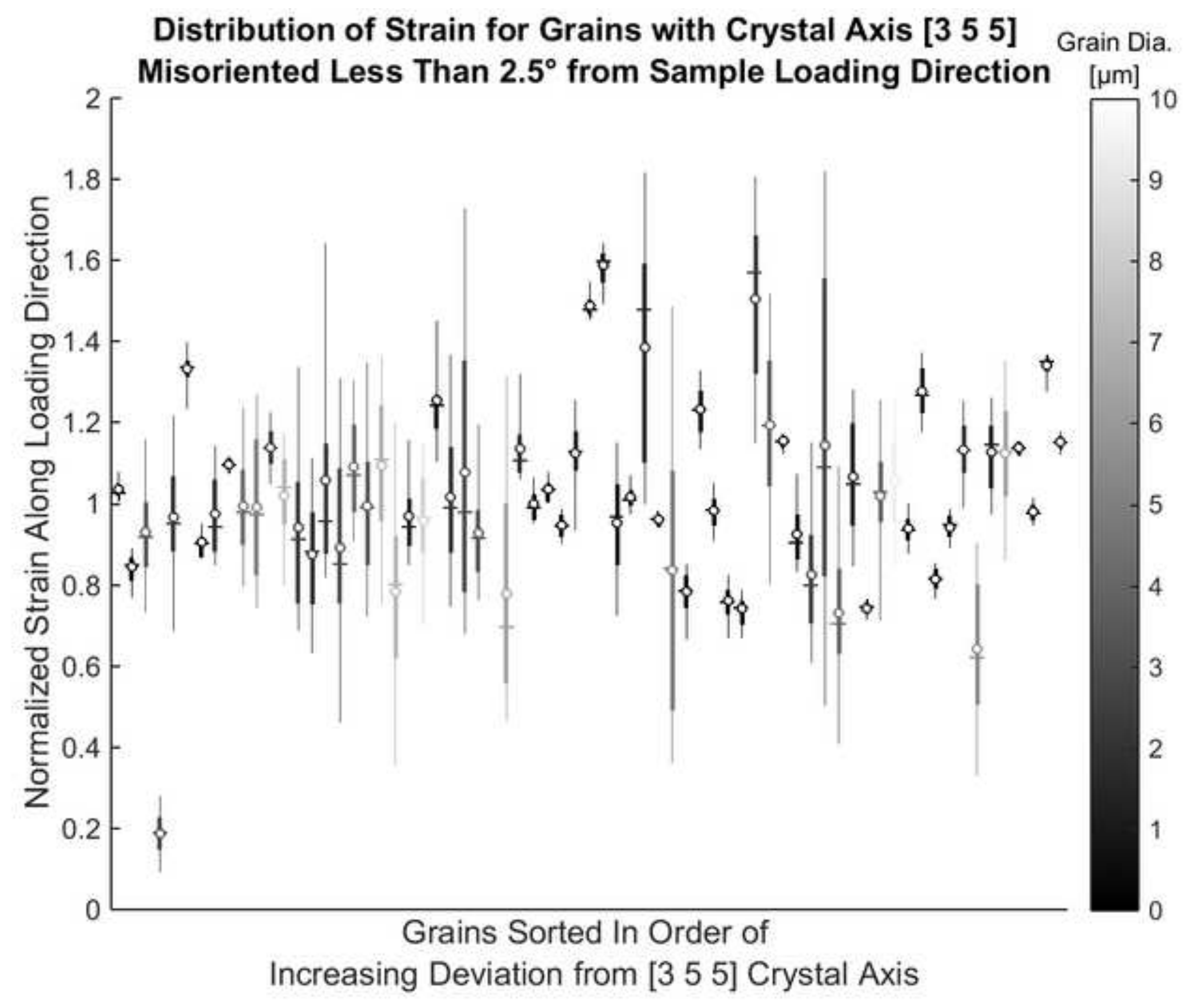




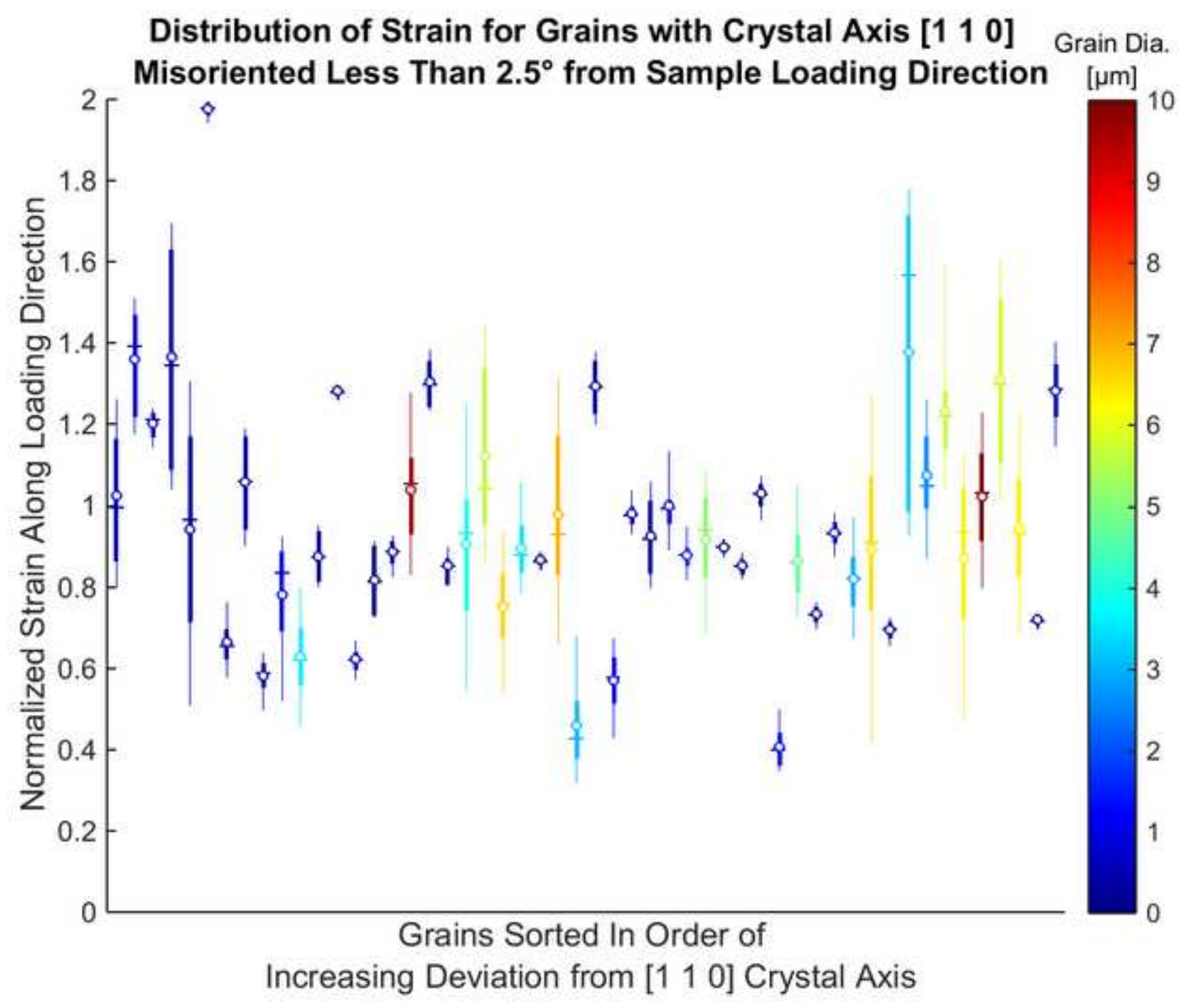




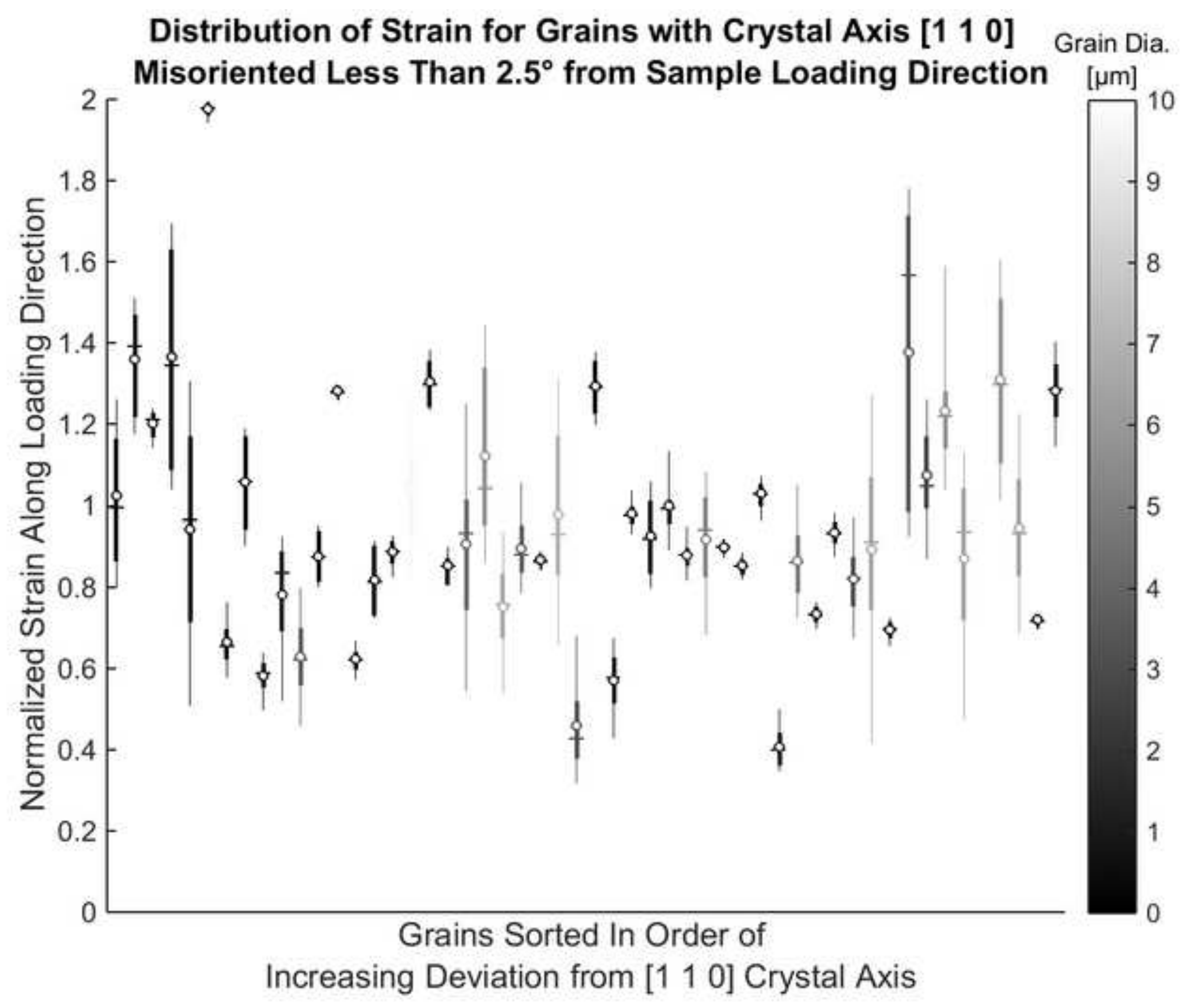


Distribution of Strain for Grains with Crystal Axis [ 1100$]$ Grain Dia.

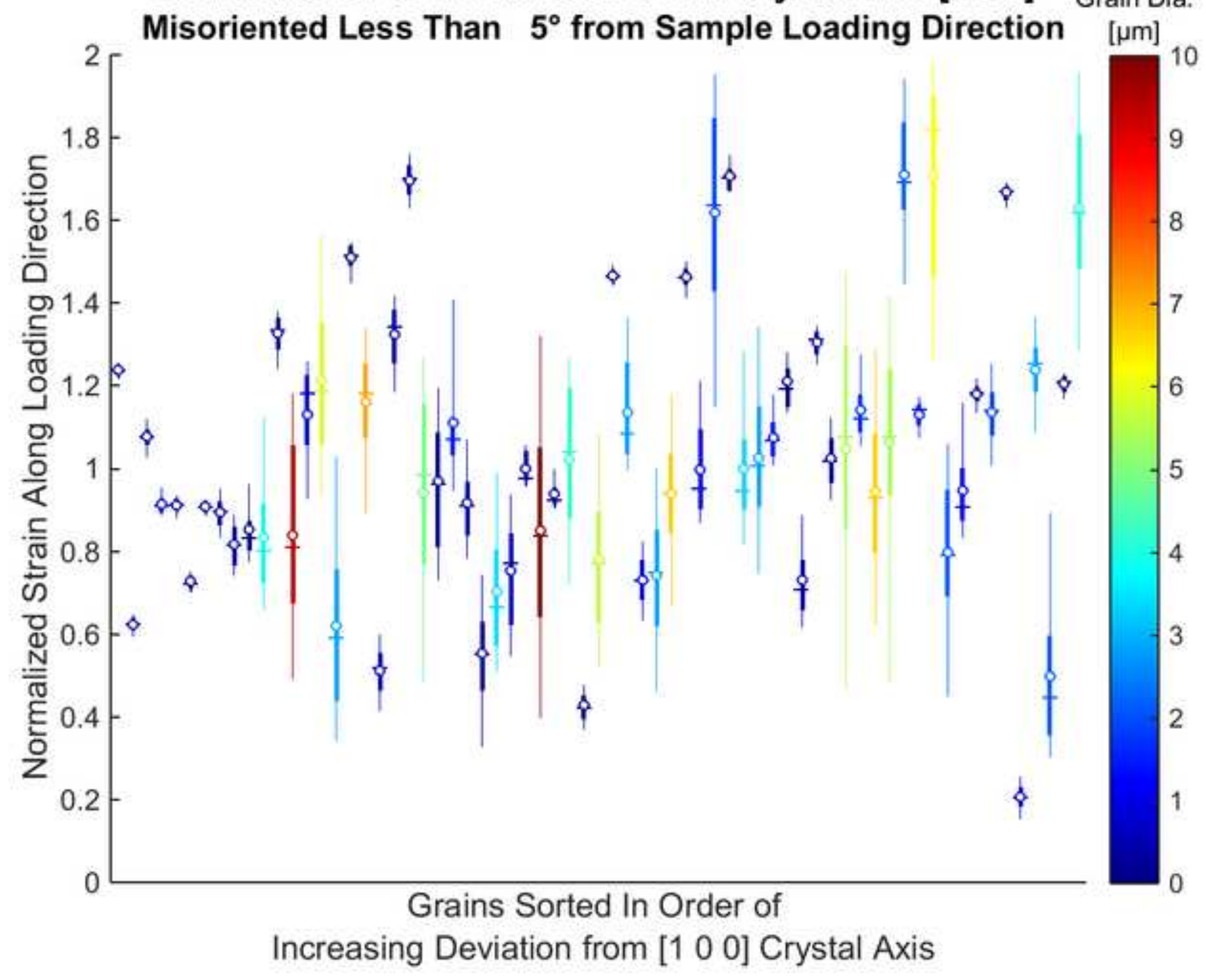


Distribution of Strain for Grains with Crystal Axis [1 00 0 $]$ Grain Dia.

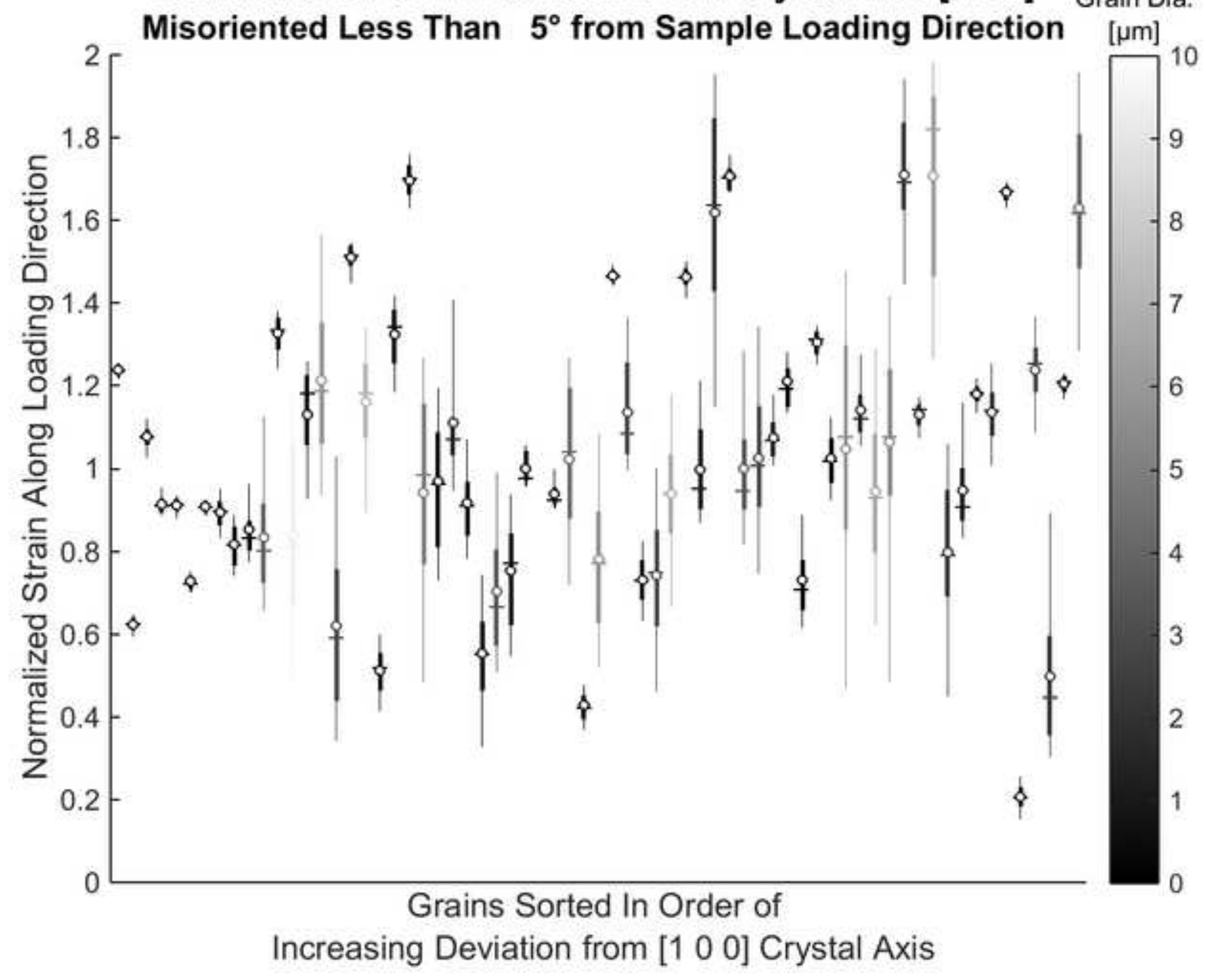


Strain Spread Distribution in All Grains

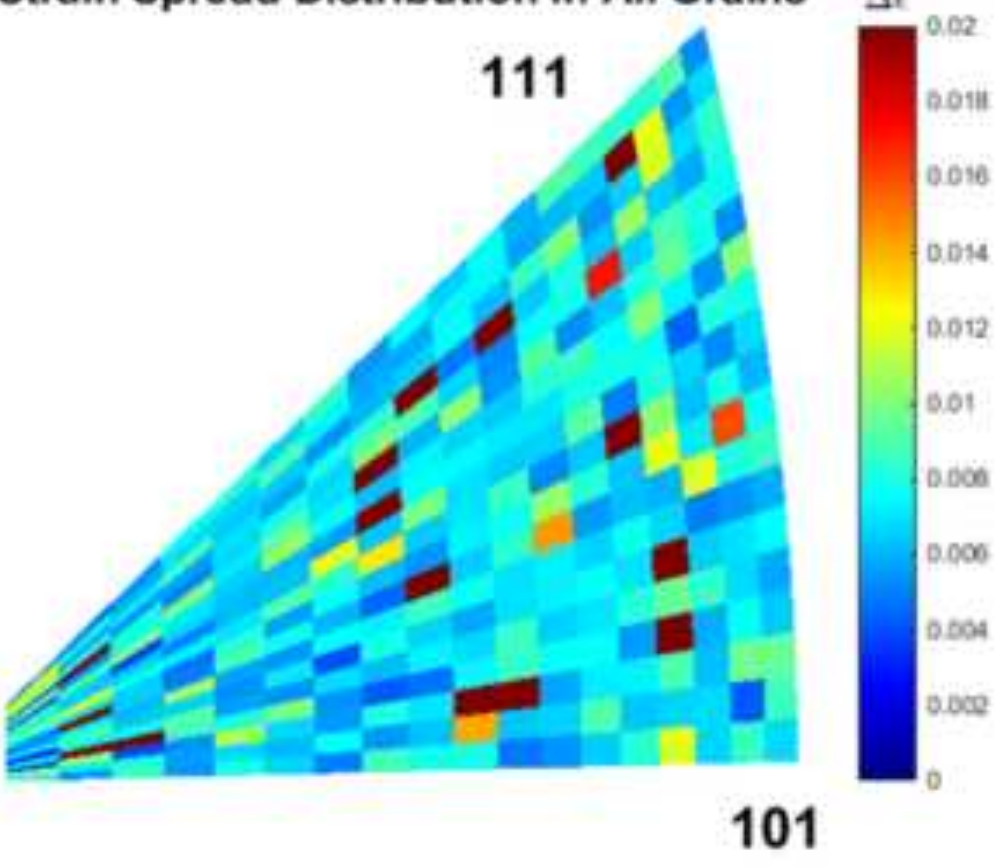

(a)

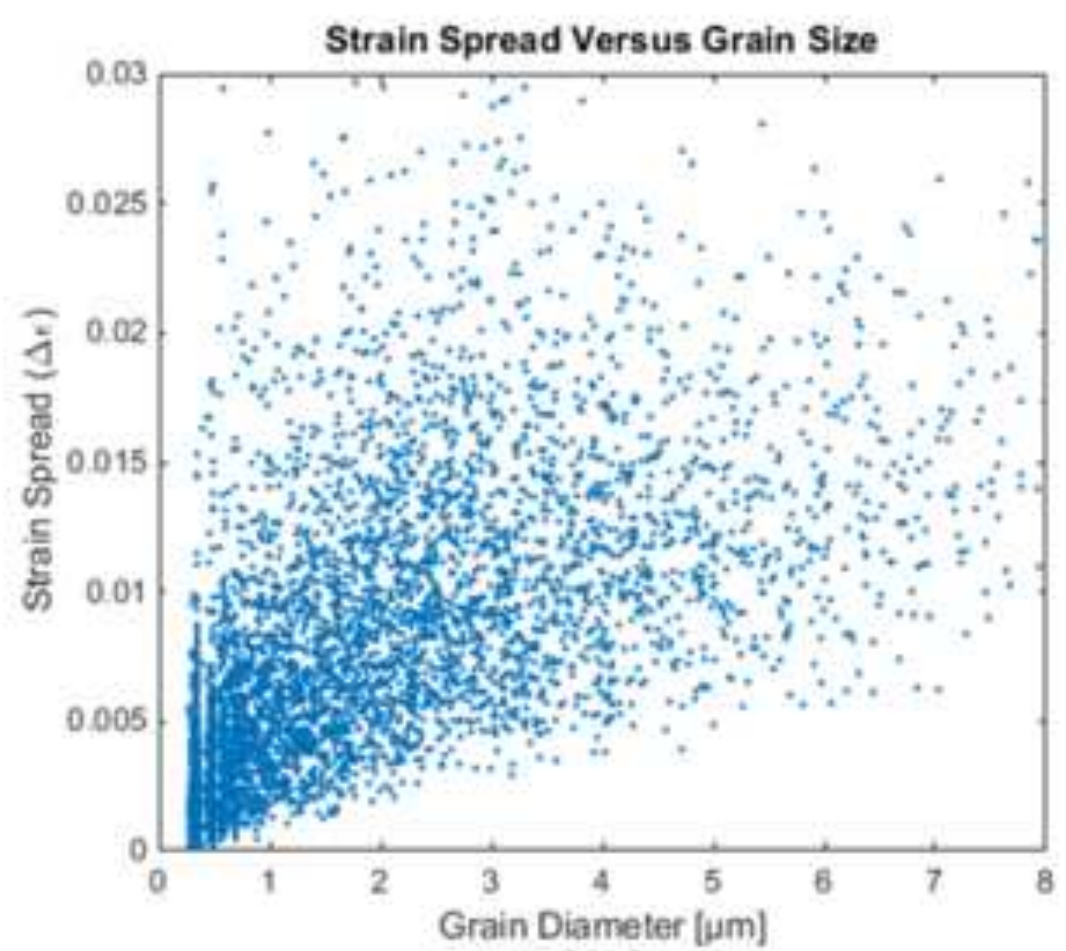

(b) 
Strain Spread Distribution in All Grains

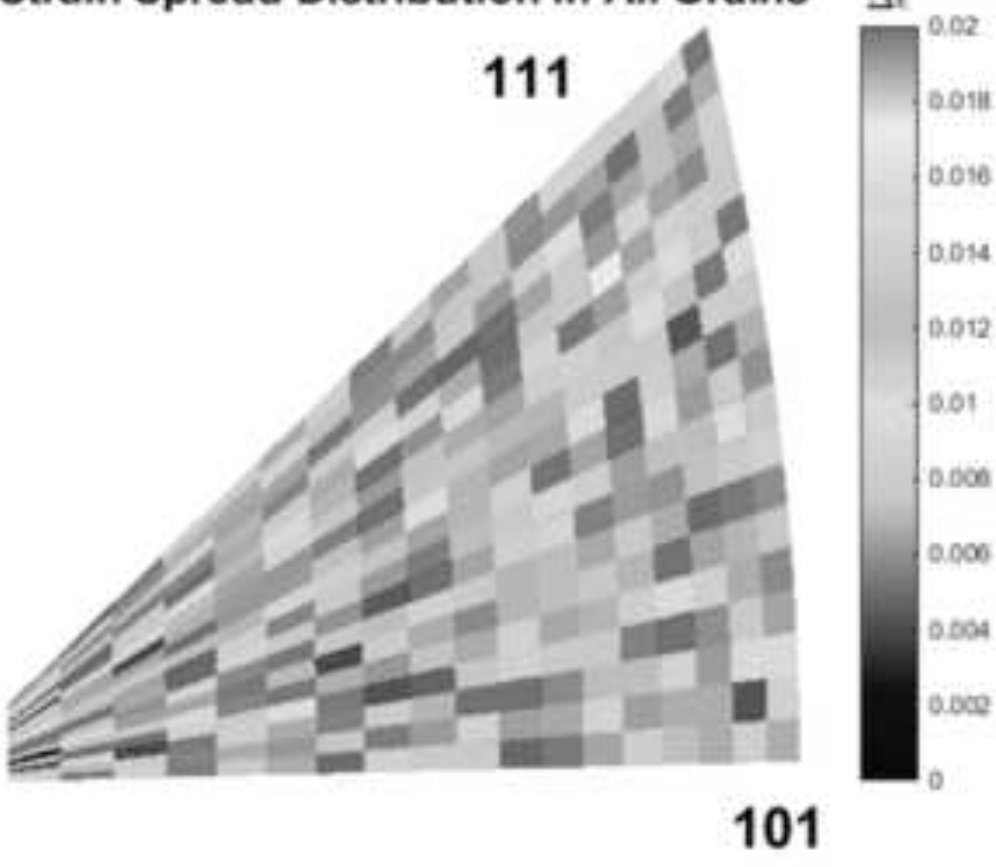

(a)

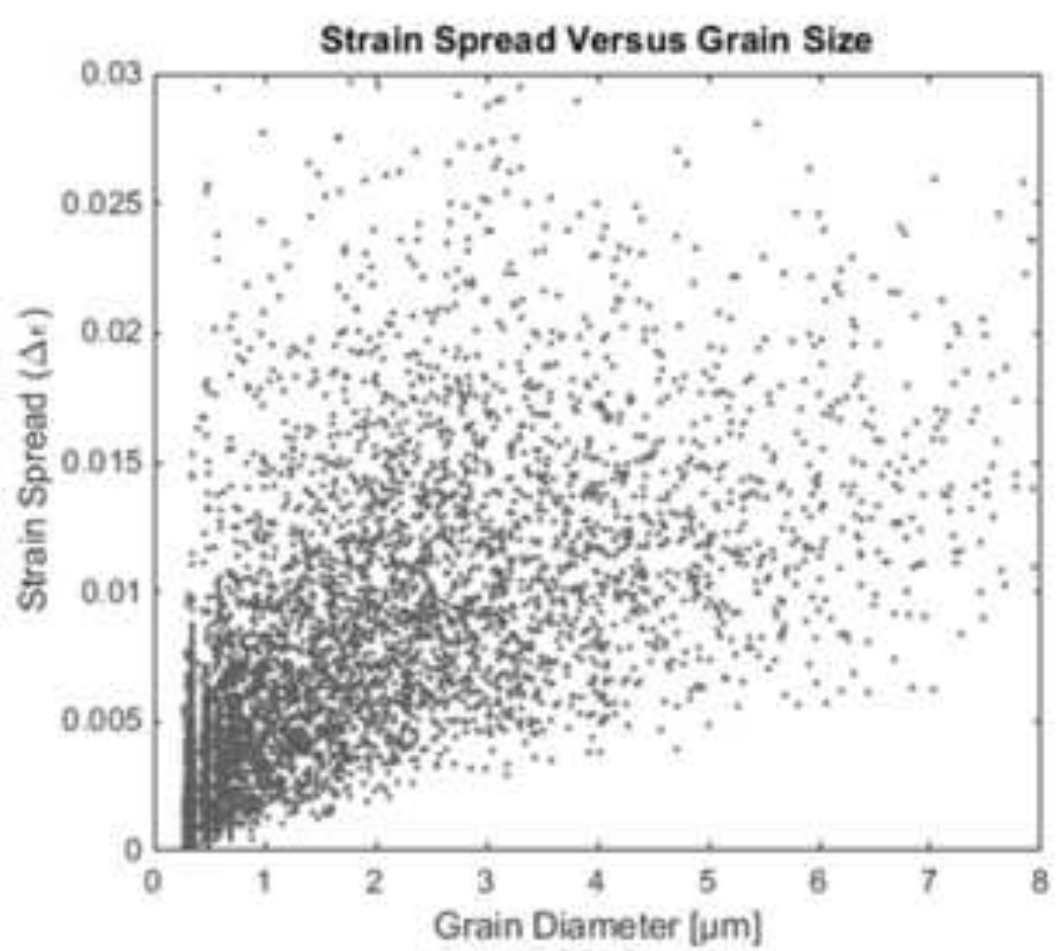

(b) 


\begin{tabular}{rccc} 
& $\begin{array}{c}\text { Predicted Transformation } \\
\text { Strain (Single Variant) }\end{array}$ & $\begin{array}{c}\text { Measured Transformation } \\
\text { Strain (Grain Averaged) }\end{array}$ & Difference \\
\hline Mean & 0.085 & 0.054 & 0.031 \\
\hline Max & 0.105 & 0.104 & 0.097 \\
\hline Min & 0.033 & 0.003 & -0.037 \\
\hline
\end{tabular}

